\title{
An Uninterpreted Spatial Version of the Trust Game: \\ Evidence of Reciprocity without Suggestive Words, Evidence of Iterated Dominance Self-Taught
}

Talbot Page and Louis Putterman

Working Paper 1/11/2012

\begin{abstract}
In this working paper we report on two trust games: a BDM-like game which is interpreted through its use of the possibly suggestive words "show up fee," "sends," "tripled," "send back"; and an uninterpreted spatial game that does not use these words suggestive or not. In the spatial game we found a considerable amount of reciprocity, which implies the words are not necessary for reciprocity.

For further comparison we designed the two games to have a correspondence relation (the relation extends to the original BDM trust game). We focused on two "variables" - interpreted or uninterpreted and spatial or word-based. We also designed "constants" which were identical or near identical in the two games. We did this to reduce confounding in statistical comparisons.

We found the frequency of reciprocity in the spatial game, without the suggestive words, was about the same as the frequency of reciprocity in the BDM-like game, with the suggestive words. We found iterated dominance in the spatial game was 5.5 times higher than in the BDM-like game. And we found sending the full endowment was significantly more frequent in the BDM-like game than in the spatial game.
\end{abstract}




\section{The Original Trust Game: Success and Open Questions}

In 1995 Berg, Dickhaut and McCabe (BDM) pioneered the trust game, opening many paths of research: definitions of trust and trustworthiness, cross-country effects, effects of markets, effects on economic growth, effects of oxytocin (for effects of oxytocin on trust see Kosfeld, Michael, Markus Heinrichs, Paul Zak, Urs Fischbacher and Ernst Fehr, 2005). In a forthcoming survey Wilson and Eckel found 84 trust game experiments, 140 unpublished papers on trust games, and 10 additional replications of the original BDM game.

But some researchers have doubts. Could the language of "send," "send back" suggest reciprocal behavior? Could these and other words be suggestive or value laden? In efforts to achieve double-blind anonymity could some subjects draw conclusions about the need for anonymity (eg."it's okay to be selfish" or "the experimenters want us to act selfishly")?

\{Pages with a blue-green background are comments and analysis; pages in Arial font are instruction slides from the experiment or individual experiments.

We thank Brown University's Department of Economics for funding the experiment reported here. We are grateful to Kenneth Ettinger, Siddharth Sastri, and Robert Franz for their assistance. We thank Ettinger, Debra Kao, Theo Page, and Iñaki Arbeloa for their supporting roles on the experimenter team. We thank Matthew Harrison for a helpful discussion of the Fisher exact test, and Chris Anderson, Kim Border, Daniel Houser, John Ledyard, Thomas Palfrey, and Charles Plott for helpful comments on a preliminary version. Talbot Page gives a special posthumous thanks to Richard McKelvey and his family. 


\section{Purpose}

The main purpose of our experiment is to take a step toward resolving the doubts or confirming them.

\section{Design}

- We designed three games. One game, a spatial game, called the Trapezoid game because of its shape, is uninterpreted and does not use the words "show up fee," "sends," "triples," "sends back."

- The second game is interpreted and its interpretation is explained by the words "show up fee," "sends," "triples," "sends back." We call this interpreted, non-spatial game "BDM-like." By design, our BDM-like game emphasizes the words more than in the original trust game.

- The third game is a hybrid with both the spatial and word-based features; its discussion is postponed for now.

- We designed "constants" which were identical or near identical in the three games.

- We designed the games to achieve a correspondence relation between them.

The next pages are instruction slides showing the large reliance on graphics in the experiment and illustrate the above designs in the game forms, double-blind procedure, correspondence relation, and the rest of the operationalization. Full instructions and practice exercises for all three games are available on the website at: http://www.econ.brown.edu/fac/Louis Putterman/instructions.html but omitted here because of time and space. 


\section{WELCOME TO AN EXPERIMENT ON DECISION MAKING}

Please sit at a desk with a folded piece of paper
taped to the desk (leave it taped for the moment)
Please do not touch the cardboard boxes in front
of you (we will use them later)

Experimenters:

Professor Talbot Page

Professor Louis Putterman

Ken Ettinger, Undergraduate

Debra Kao, Undergraduate Theo Page

Brown University

November 14, 2009 


\section{Monitors}

We start by selecting 2 monitors (the selection depends on what seat you chose). The monitors will verify that the instructions are exactly followed and will be in charge of the information flow, as explained below.

The monitors have a responsible and essential role, as will become clear in the experiment. Each monitor will be paid $\$ 20$.

Now that everyone is seated, untape the paper on your desk. If the letter A or $\mathrm{C}$ is written on the other side of the paper, you have been selected to be a monitor. 


\section{INSTRUCTIONS}

Please listen carefully to the instructions, which are to help you understand the experiment. In the experiment itself, you will be reminded of each step, but with less explanation. If you have questions of clarification, at the end of the instructions raise your hand, and we will come to your desk to answer your questions.

We ask that you do not talk, at all. Please turn off cell phones, computers and other electronic devices. 
Participants who are seated on this side of the room will have the role of "Red" and will be referred to as "Red" or "Reds." Each Red will be given a yellow highlighter.

Participants who are seated on this side of the room will have the role of "Blue" and will be referred to as "Blue" or "Blues." Each Blue will be given a black ink pen.

Each Red participant will be randomly paired with a Blue participant (and each Blue participant will be paired with a Red participant).

You won't know the identity of the person you will be paired with, and the other person won't know your identity, either.

\section{In this experiment you will make one single decision and the person you will be paired with will make one single decision.}

The two decisions will determine a location in a diagram. The location will determine your payment and the payment to the person you are paired with. \{Subjects in the Trapezoid game read the above 2 sentences and the next 7 slides.\} 


\section{The Diagram and Possible Locations}

The diagram is a trapezoid with a green triangle in it. The dots are possible locations. The possible locations are intersections of the grid in the green triangle or on its boundary.

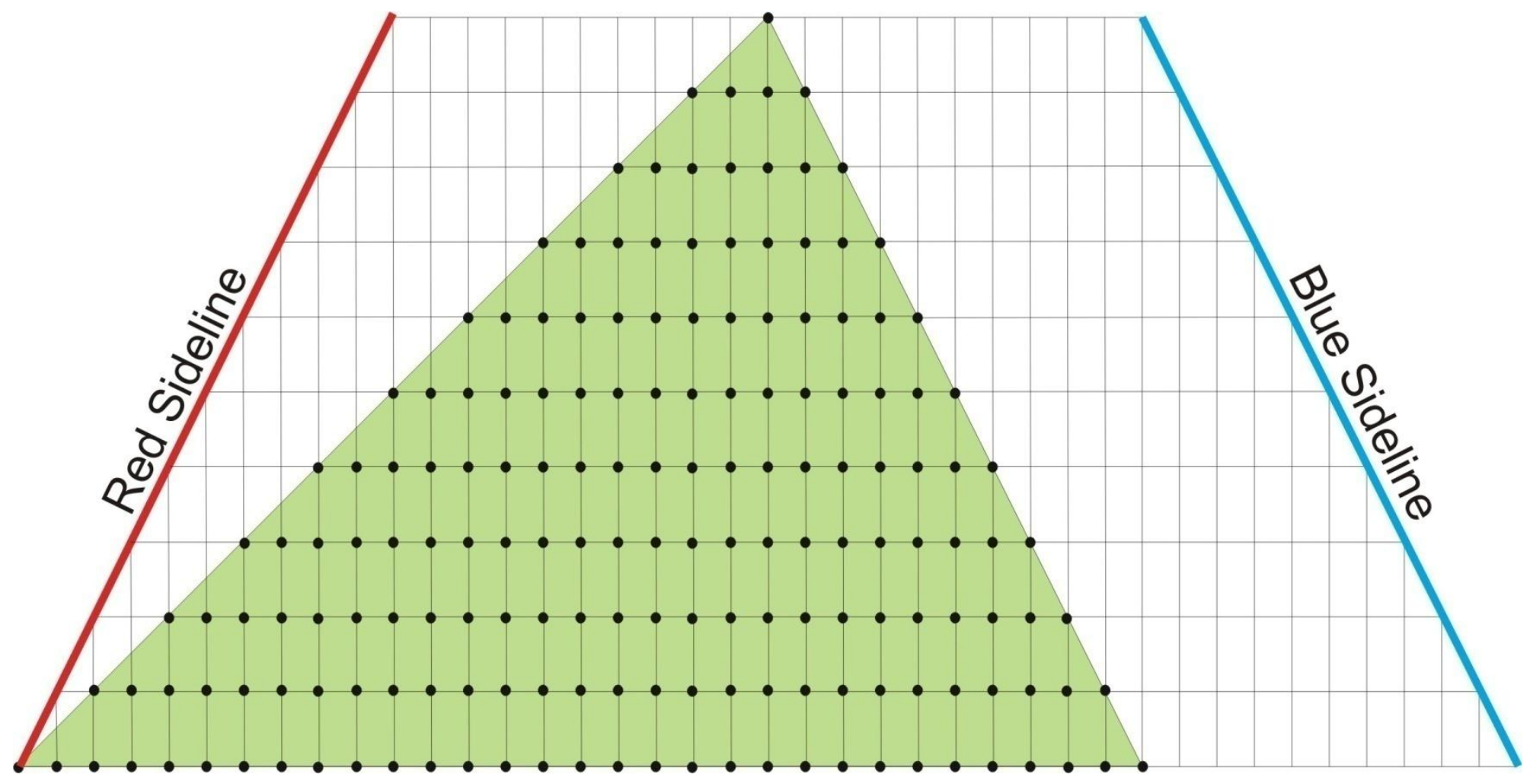

Red will make a decision on how high or low the location will be.

Blue will make a decision on how far to the left or right the location will be in the triangle. The two decisions determine the location. 


\section{Red's Decision}

Red can choose any horizontal grid line. It could be the top grid line, the bottom grid line, or any of the other grid lines in between.
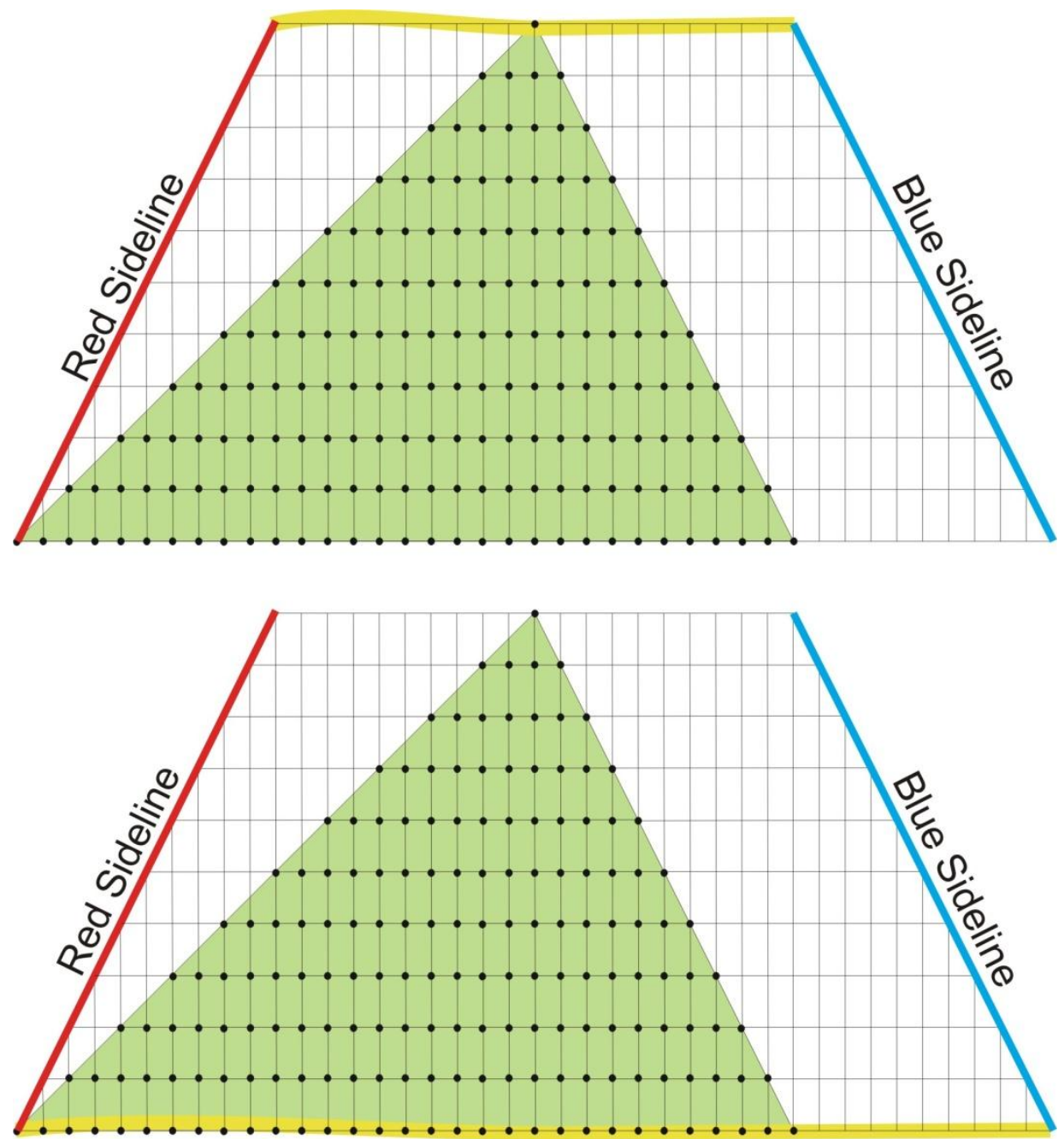


\section{Red's Decision}

In this example, Red chooses the line as shown below. Red indicates his or her choice by highlighting the chosen line with a yellow highlighter.

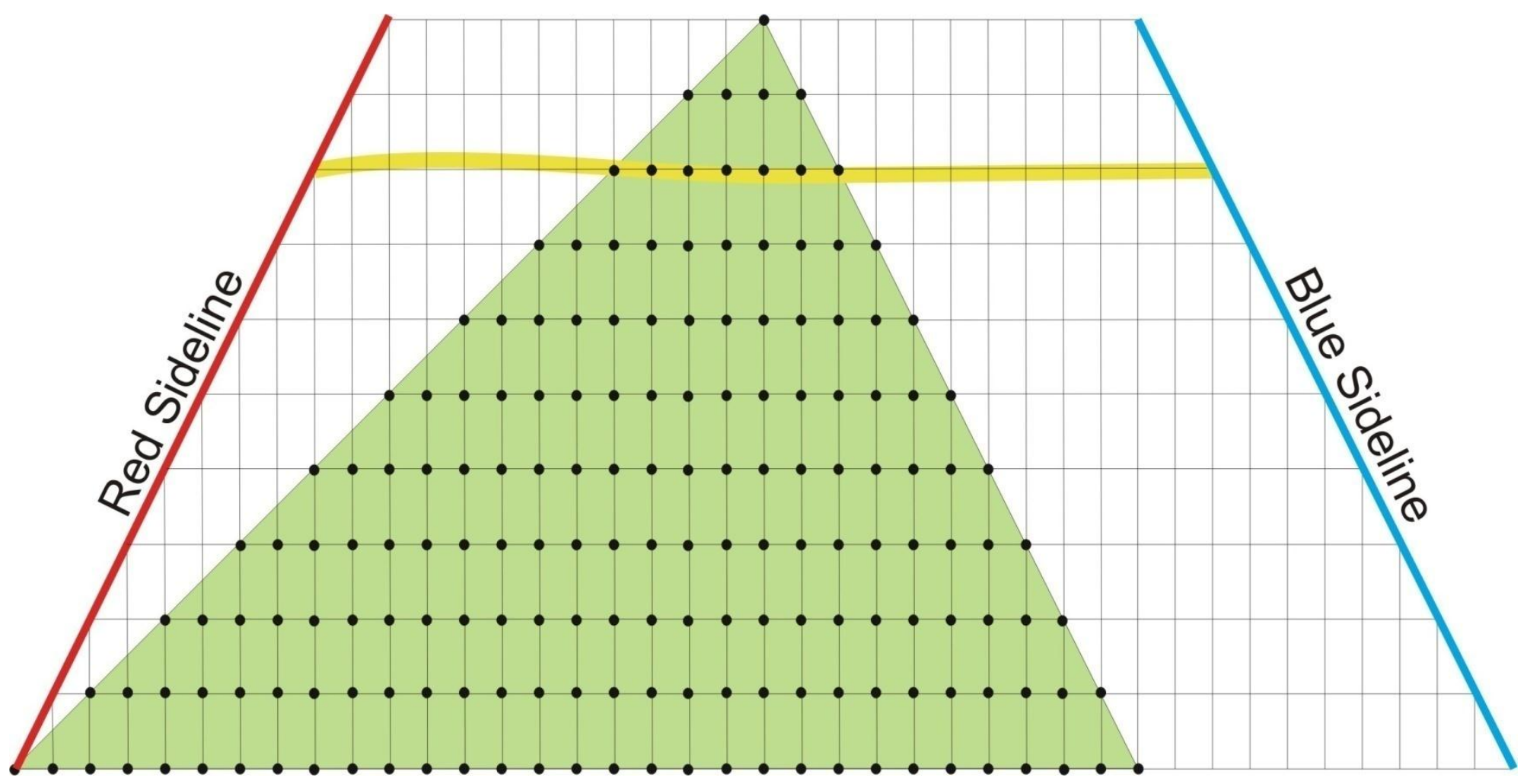

The above is only an example. The actual decision is made by each person. 


\section{Blue's Decision}

Blue can choose any dot on the yellow line that Red chose. On the yellow line, Blue can choose the left most dot, the right most dot, or any dot in between. Note there are no dots outside of the green triangle.
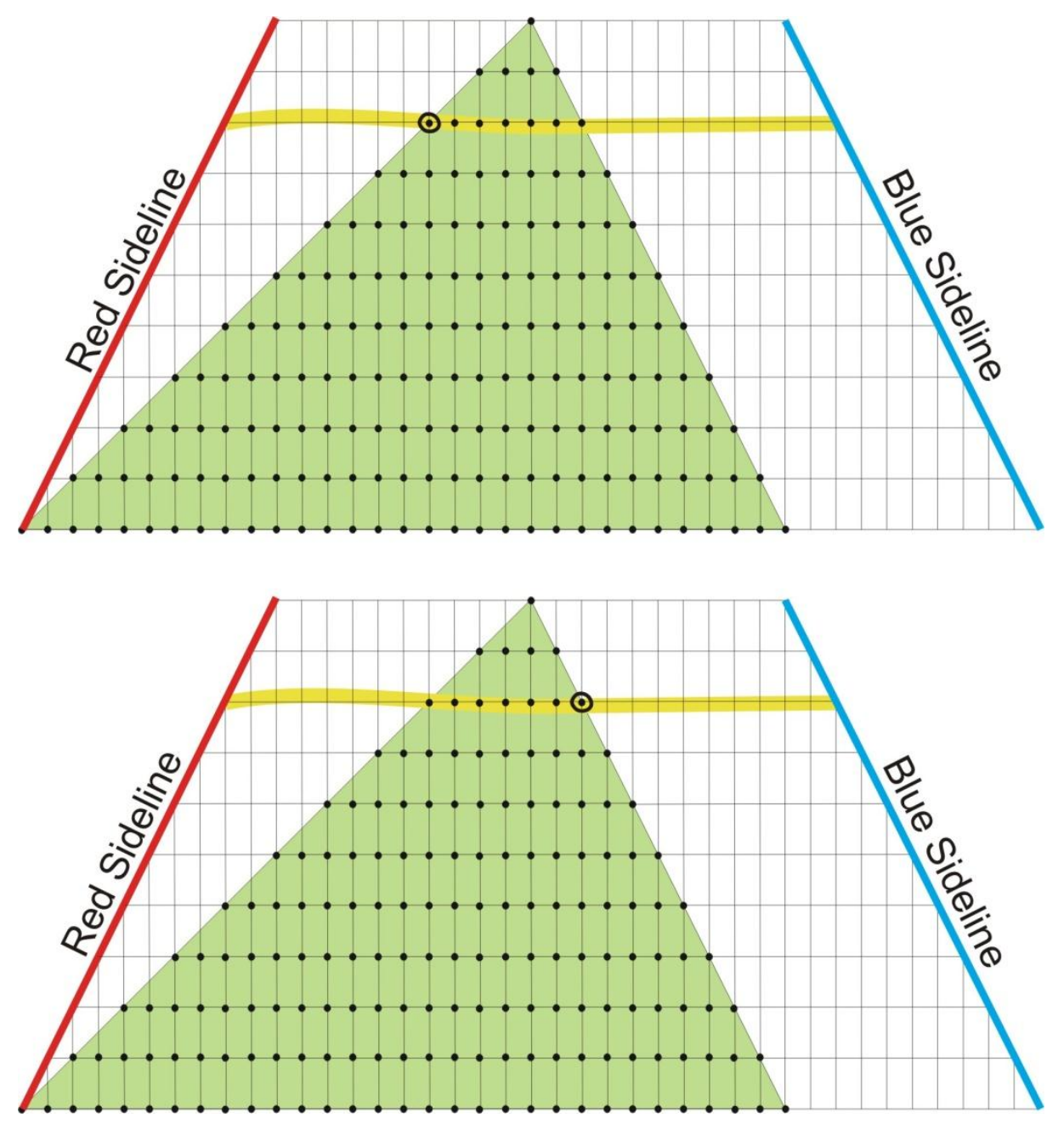


\section{Blue's Decision}

Continuing the example, Blue's chooses the dot as shown below. Blue indicates her or his decision by using a black ink pen to circle the chosen dot.

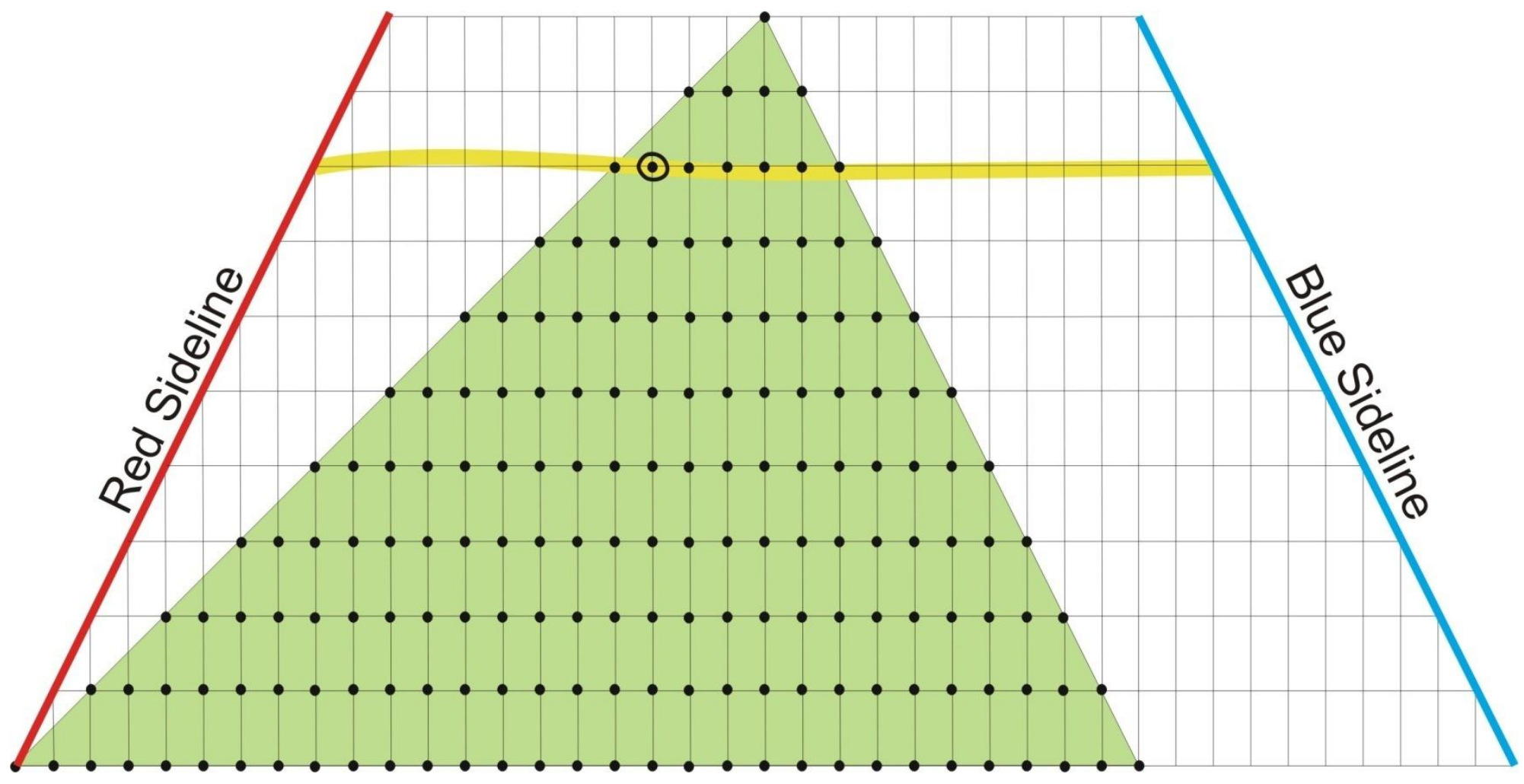

Red's and Blue's decisions together determine the chosen location, The location is indicated by the circled dot in the example. 


\section{Payments}

If you are Red, your payment is the horizontal distance from the chosen location to the Red Sideline.

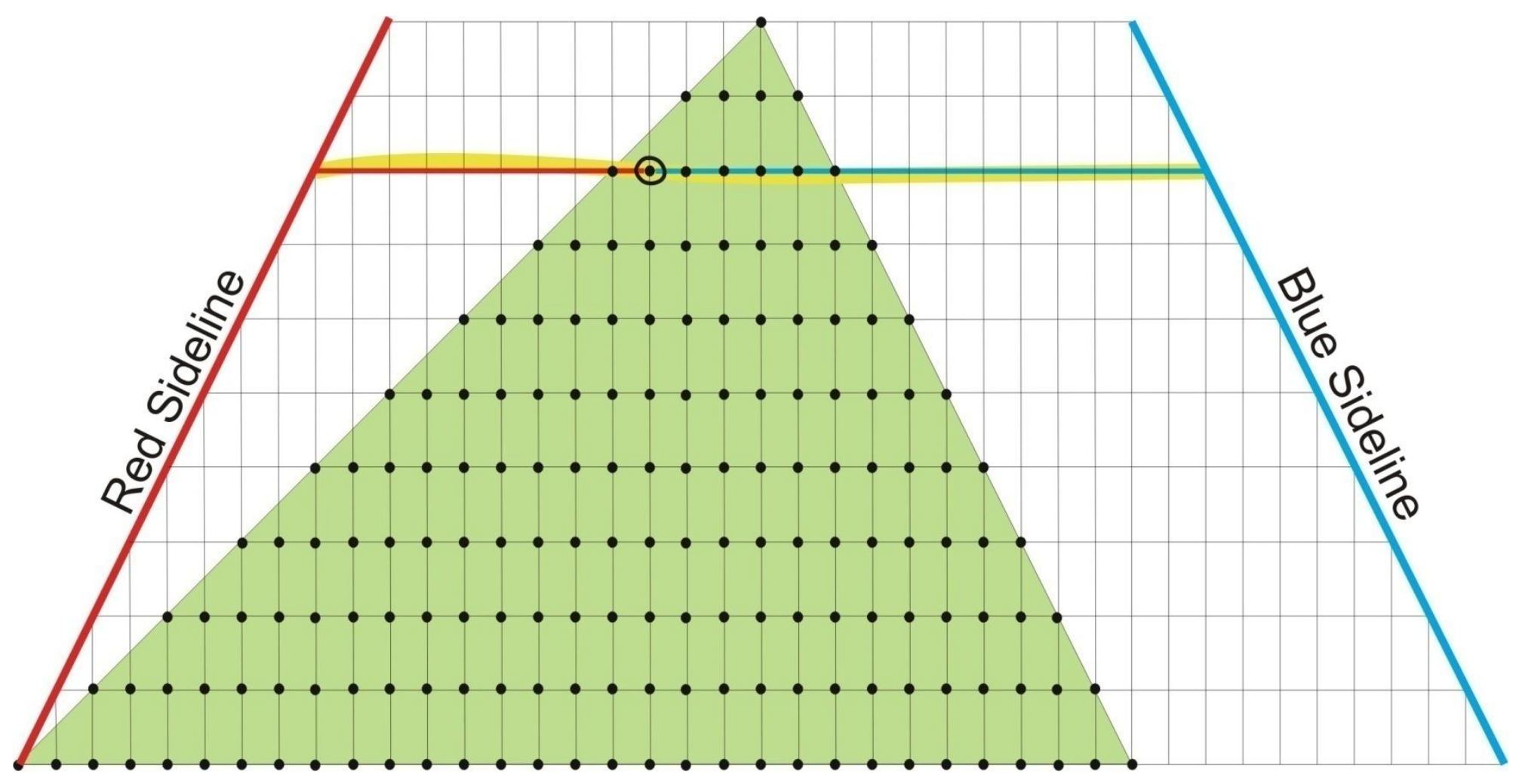

If you are Blue, your payment is the horizontal distance from the chosen location to the Blue Sideline. 


\section{Payments}

The horizontal distances are measured in grid widths. In the example there are 9 grid widths from the chosen dot to Red's Sideline. Each grid width is worth $\$ 1$. So in the example Red's payment is $\$ 9$.

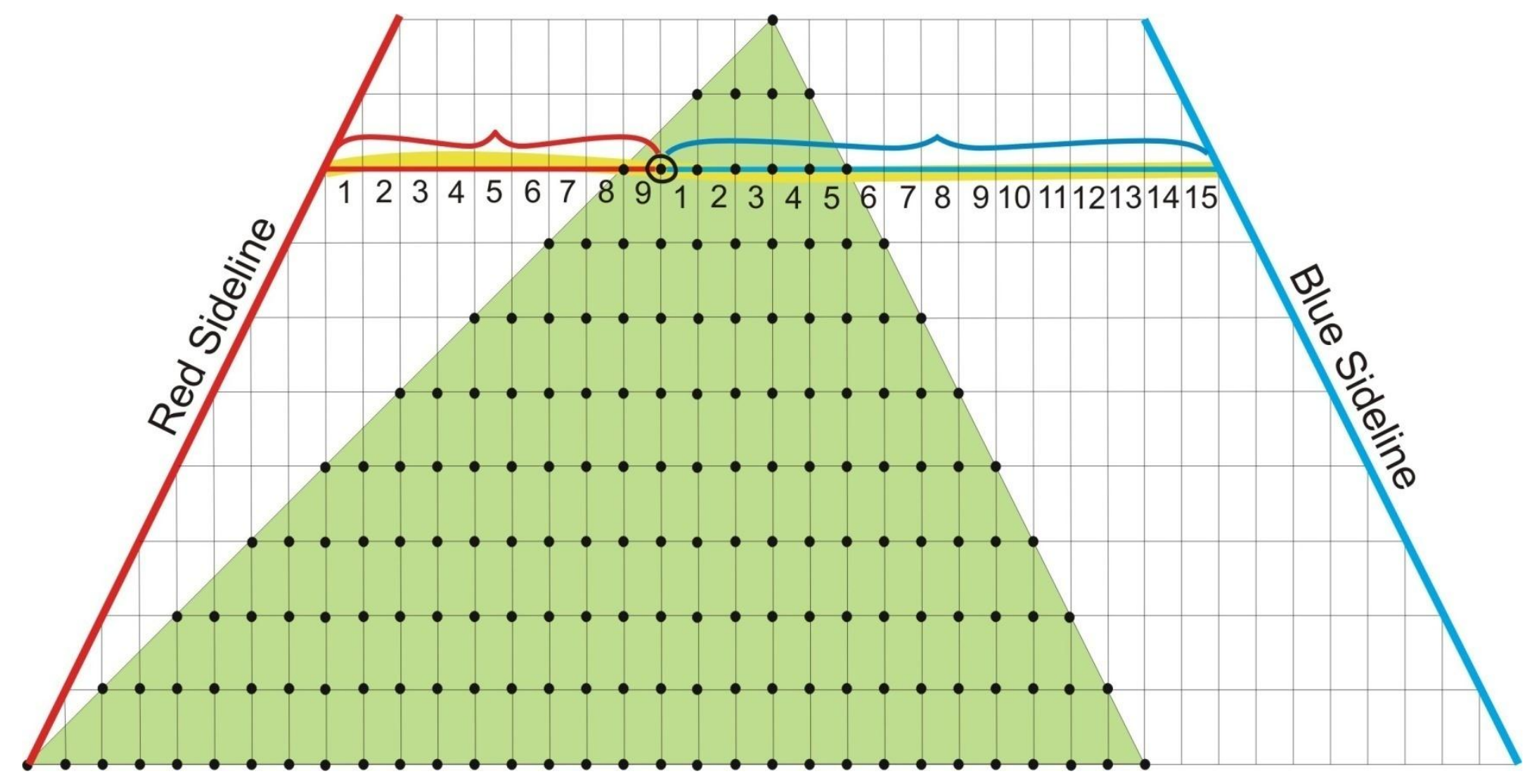

In the example there are15 grid widths from the chosen dot to Blue's Sideline, and Blue's payment is $\$ 15$. 
In the above example from the uninterpreted Trapezoid game Red's action was to choose the third line from the top and Blue's action was to circle the second dot to the left. With these decisions Red's payment was $\$ 9$ and Blue's payment was $\$ 15$.

Next we consider the interpreted BDM-like's game form and an example from the BDM-like game.

$\{$ No subject participated in more than one game or session. Subjects in the BDM-like game read the next 7 slides. \} 


\section{Show-Up Fees and Decisions}

Each Red and Blue participant is given $\$ 10$ as a show-up fee.

Each Red participant is given the opportunity to send some, all, or none of his/her show-up fee to an anonymous Blue participant.

The experimenter matches, twice over, the amount Red sends, and Blue receives the tripled amount.

Then Blue is given the opportunity to send back to the anonymous Red participant some, all, or none of the amount that Blue received. 


\section{The Decision Sheet}

Red and Blue indicate their decisions on a decision sheet, as shown below.

The amount of Red's $\$ 10$ show-up fee Red sends to Blue

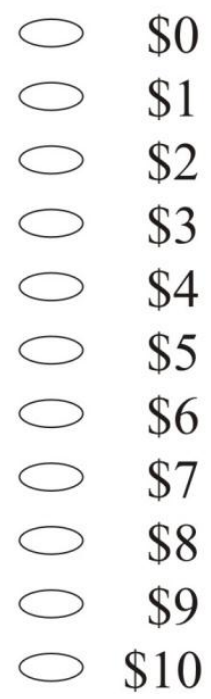

The amount is tripled and Blue receives this tripled amount

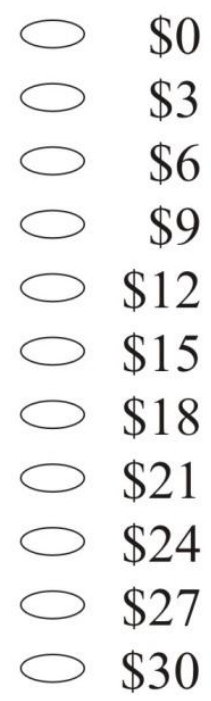

The amount Blue sends back to Red

$\begin{array}{llllll}0 & \$ 0 & 0 & \$ 0 & 0 & \$ 0 \\ 0 & \$ 1 & 0 & \$ 2 & 0 & \$ 3 \\ 0 & \$ 4 & 0 & \$ 5 & 0 & \$ 6 \\ 0 & \$ 7 & 0 & \$ 8 & 0 & \$ 9 \\ 0 & \$ 10 & 0 & \$ 11 & 0 & \$ 12 \\ 0 & \$ 13 & 0 & \$ 14 & 0 & \$ 15 \\ 0 & \$ 16 & 0 & \$ 17 & 0 & \$ 18 \\ 0 & \$ 19 & 0 & \$ 20 & 0 & \$ 21 \\ 0 & \$ 22 & 0 & \$ 23 & 0 & \$ 24 \\ 0 & \$ 25 & 0 & \$ 26 & 0 & \$ 27 \\ 0 & \$ 28 & 0 & \$ 29 & 0 & \$ 30\end{array}$




\section{An Example: Red's Decision}

As an example, Red decides to send $\$ 2$ to Blue (and Red keeps the remaining $\$ 8$ of the show-up fee). Red fills in the $\$ 2$ bubble.

The amount of Red's $\$ 10$ show-up fee Red sends to Blue

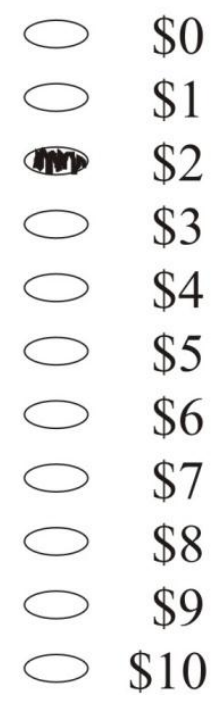

The amount is tripled and Blue receives this tripled amount
The amount Blue sends back to Red

$\begin{array}{llllllll}0 & \$ 0 & 0 & \$ 0 & 0 & \$ 0 & 0 & \$ 0 \\ 0 & \$ 3 & 0 & \$ 1 & 0 & \$ 2 & 0 & \$ 3 \\ 0 & \$ 6 & 0 & \$ 4 & 0 & \$ 5 & 0 & \$ 6 \\ 0 & \$ 9 & 0 & \$ 7 & 0 & \$ 8 & 0 & \$ 9 \\ 0 & \$ 12 & 0 & \$ 10 & 0 & \$ 11 & 0 & \$ 12 \\ 0 & \$ 15 & 0 & \$ 13 & 0 & \$ 14 & 0 & \$ 15 \\ 0 & \$ 18 & 0 & \$ 16 & 0 & \$ 17 & 0 & \$ 18 \\ 0 & \$ 21 & 0 & \$ 19 & 0 & \$ 20 & 0 & \$ 21 \\ 0 & \$ 24 & 0 & \$ 22 & 0 & \$ 23 & 0 & \$ 24 \\ 0 & \$ 27 & 0 & \$ 25 & 0 & \$ 26 & 0 & \$ 27 \\ 0 & \$ 30 & 0 & \$ 28 & 0 & \$ 29 & 0 & \$ 30\end{array}$




\section{Continuing the Example: The Experimenter Triples}

The experimenter triples the $\$ 2$ to $\$ 6$ and Blue receives the $\$ 6$. The experimenter fills in the $\$ 6$ bubble.

The amount of Red's $\$ 10$ show-up fee Red sends to Blue

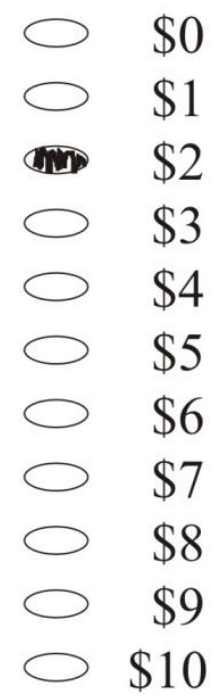

The amount is tripled and Blue receives this tripled amount
The amount Blue sends back to Red
$\bigcirc \$ 0$
○ $\$ 3$
- $\$ 6$
$\bigcirc \$ 9$
- $\$ 12$
○ $\$ 15$
- $\$ 18$
○ $\$ 21$
○ $\$ 24$
○ $\$ 27$
○ $\$ 30$

- $\$ 0$

○ $\$ 0$

- $\$ 0$ 


\section{Continuing the Example: Experimenter Shows Limit}

Blue cannot send back more than the amount that Blue received. The experimenter shows this limit by drawing the crossed-out rectangle.

The amount of Red's $\$ 10$ show-up fee Red sends to Blue

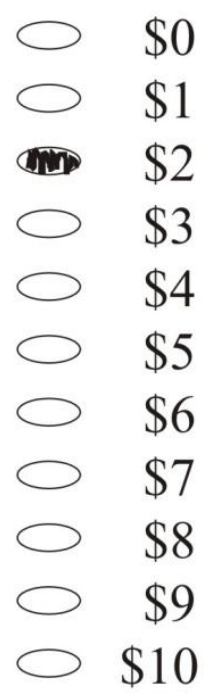

The amount is tripled and Blue receives this tripled amount

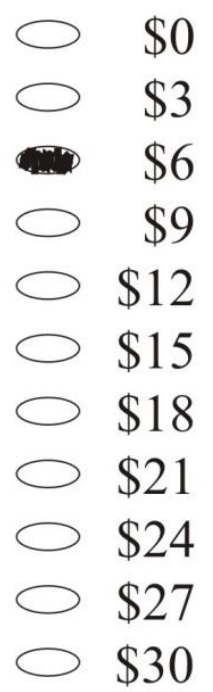

The amount Blue sends back to Red

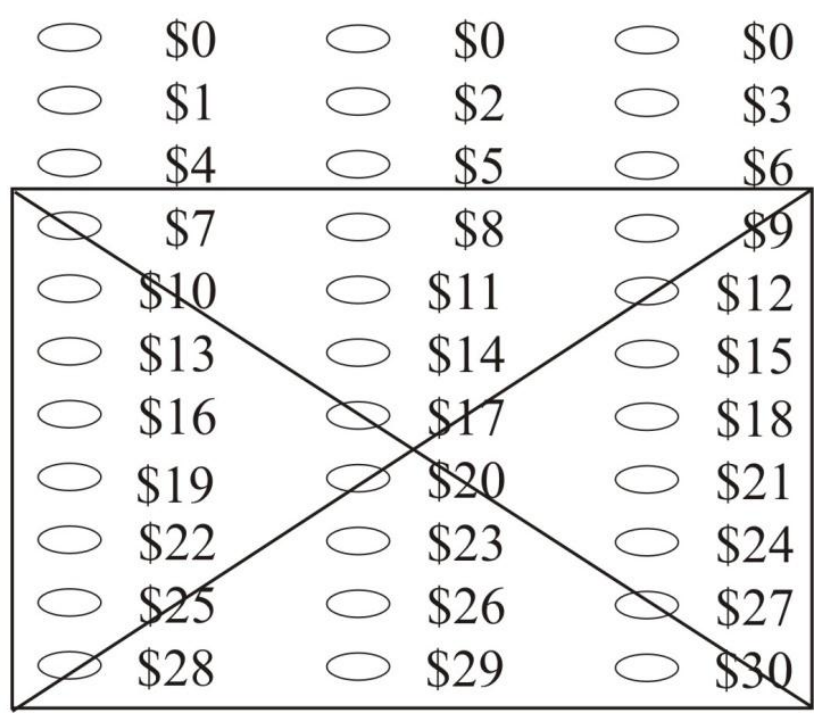




\section{Continuing the Example: Blue's Decision}

In the example, Blue decides to send back to Red $\$ 1$ (and Blue keeps the remaining $\$ 5$ of the $\$ 6$ amount Blue received). Blue fills in the $\$ 1$ bubble.

The amount of Red's $\$ 10$ show-up fee Red sends to Blue

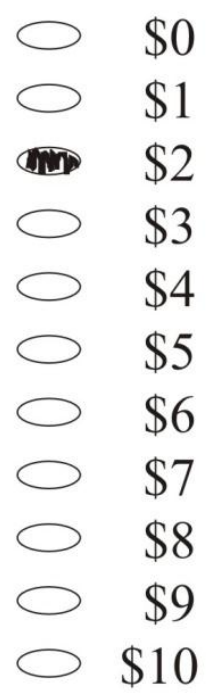

The amount is tripled and Blue receives this tripled amount

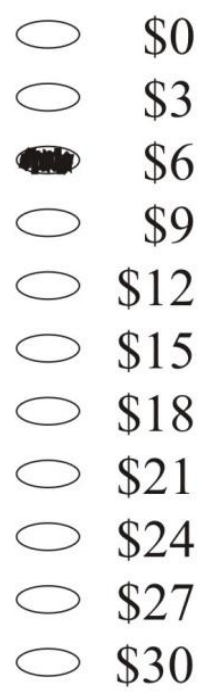

The amount Blue sends back to Red

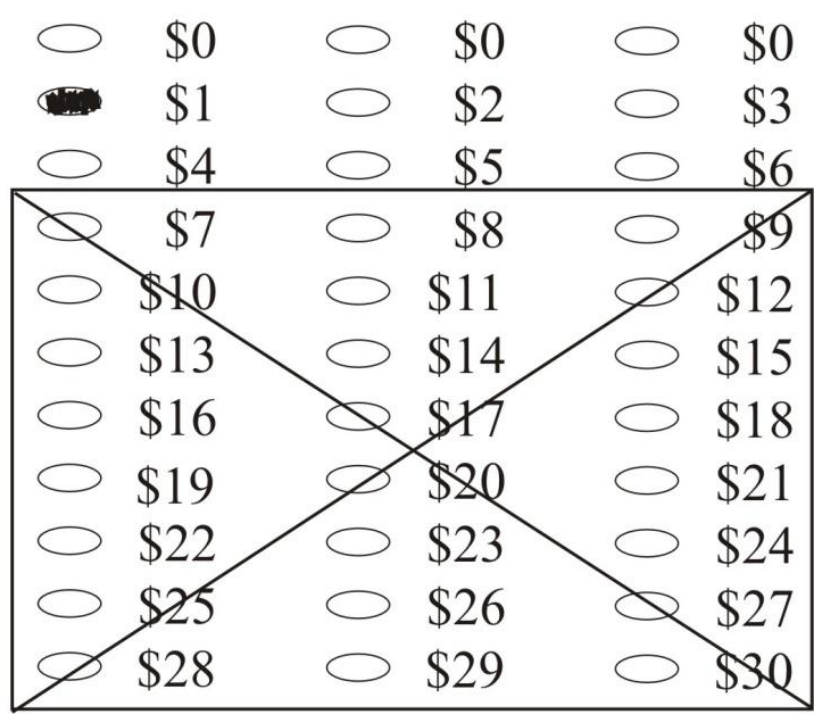




\section{The Example Continued: The Money Outcomes}

The amount of Red's $\$ 10$ show-up fee Red sends to Blue
The amount is tripled and Blue receives this tripled amount

$\begin{array}{rr}0 & \$ 0 \\ 0 & \$ 1 \\ & \$ 2 \\ 0 & \$ 3 \\ 0 & \$ 4 \\ 0 & \$ 5 \\ 0 & \$ 6 \\ 0 & \$ 7 \\ 0 & \$ 8 \\ 0 & \$ 9 \\ 0 & \$ 10\end{array}$

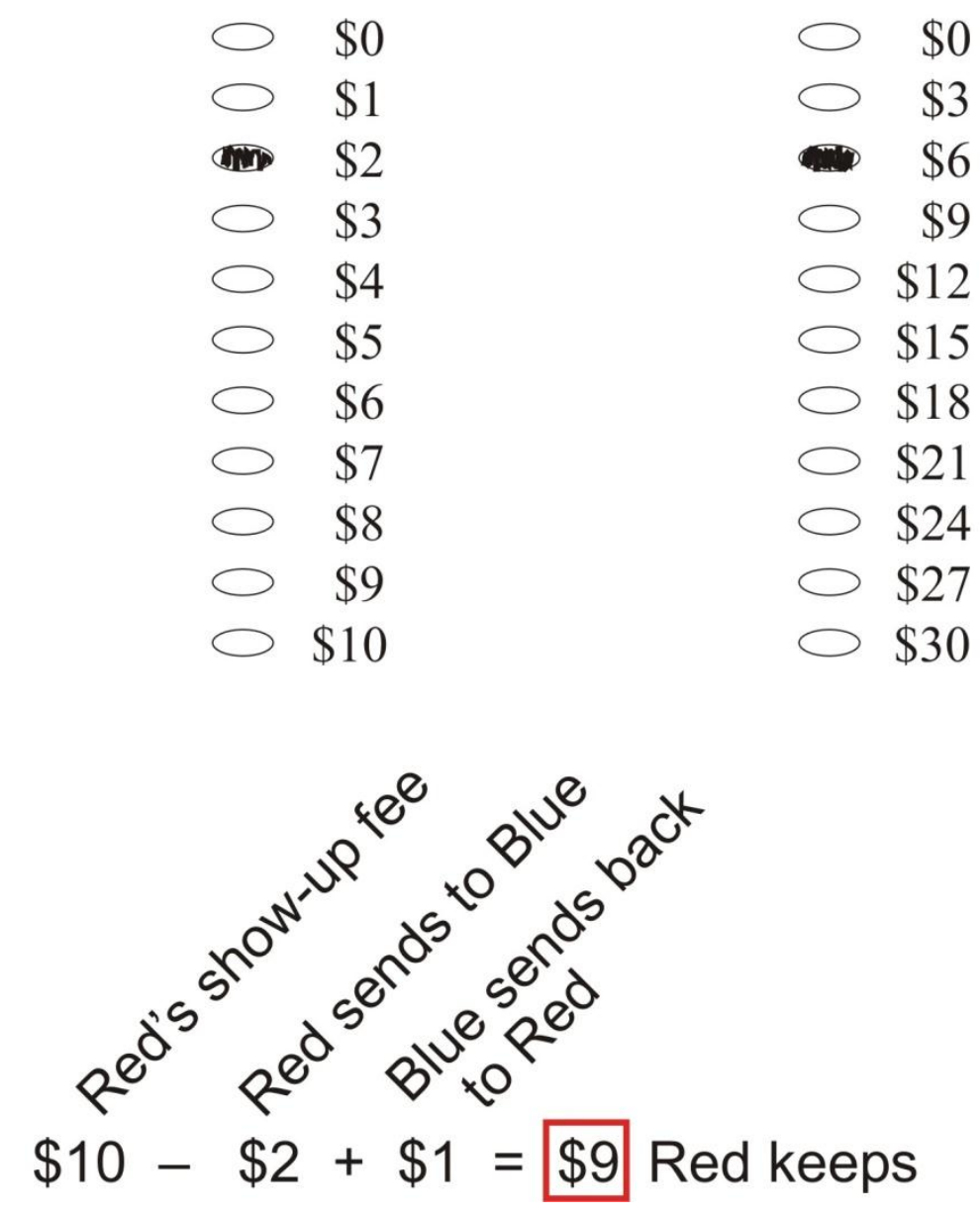

The amount Blue sends back to Red
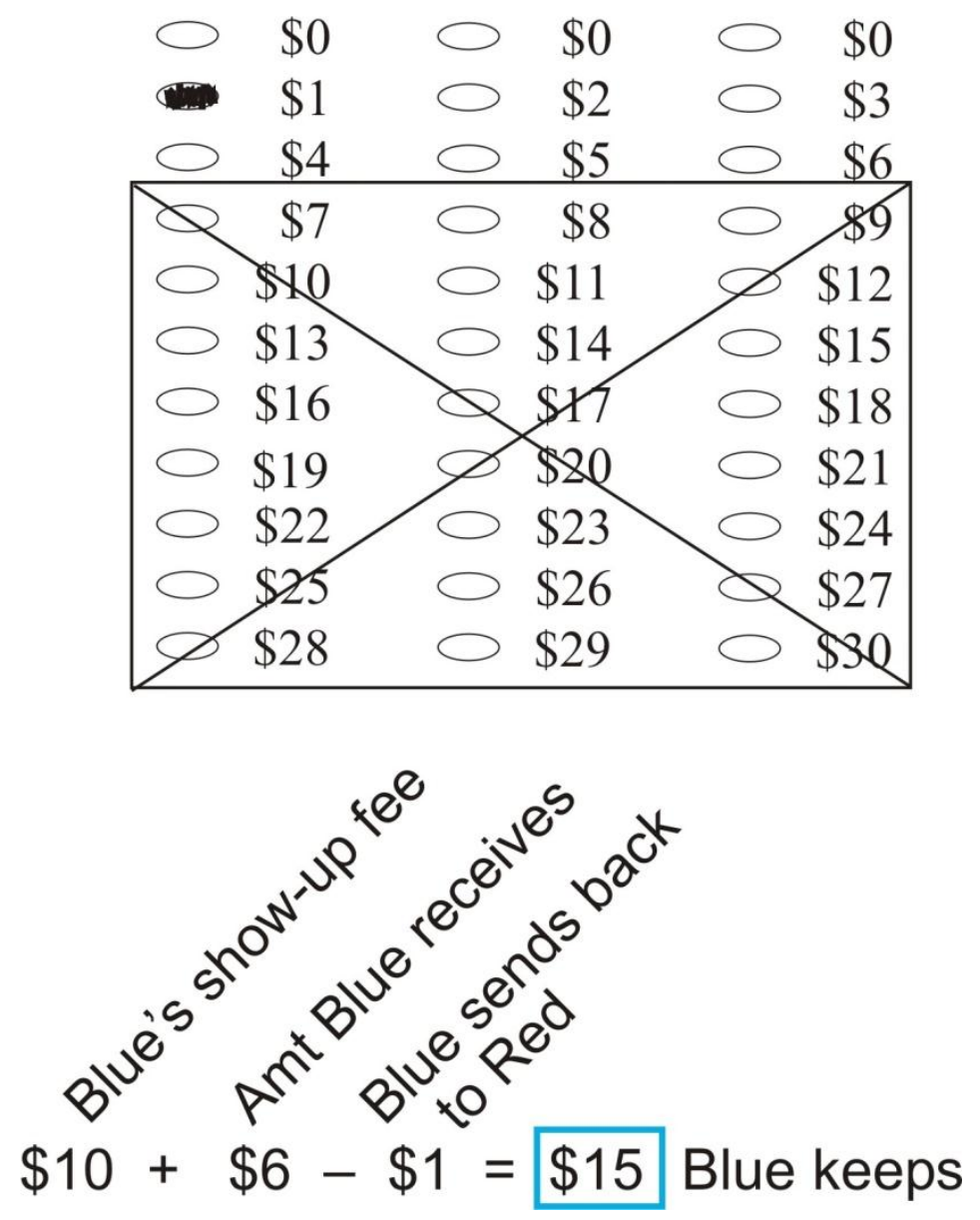
In the BDM-like game Red's action was to send \$2 to Blue and Blue's action was to send back $\$ 1$.

In the Trapezoid example Red's action was to choose a line and Blue's action was to choose a dot.

The actions differ but Red's payment of $\$ 9$ was the same in the Trapezoid example as it was in the BDM-like example. Similarly, Blue's payment of \$15 was the same in the two games.

The two games differ, one uninterpreted the other interpreted, and differ between spatial or word-based forms. The examples show that the subjects' actions can vary but have the same payments.

\{The next 3 slides are nearly identical in both the Trapezoid and BDM-like games and illustrate the constancy. 


\section{Privacy}

This is a "double blind" experiment.

We have structured the experiment so that the experimenters and monitors will not learn who made what decision. Following a fairly simple procedure we will be able to make the correct payments without knowing your identity as a decision maker.

To make your and others' decisions private we ask you and others:

(1) Not to tell your decision to others

(2) To take care to block the view of your highlight or circle* from others when you make your decision.

If you and others follow these steps, you will experience an experiment on private decision making.

"In the BDM-like game, the words "highlight or circle" are replaced by "decision sheet." 


\section{Claim Checks}

In this experiment, getting the correct payments while preserving privacy is made possible by using "claim checks." Here is the idea:

If you go to a museum, you may give your coat to a "coat check" person. The check person sometimes has specially printed tickets, each with a number at the top, the same number at the bottom, and perforated in the middle. The check person tears a ticket and gives you the top half of it and keeps the bottom half, which is attached to your coat. When you are ready to leave, you give your half of the ticket to the check person. The check person finds the coat with the matching number on the bottom half of the ticket, and gives you your coat. There is no confusion over which coat to return, because the matching number on each half of your ticket differs from the number on any other ticket. 


\section{Claim Checks}

The claim checks in this experiment differ from the example in some ways:

First, a claim check is not made up by a ticket torn in two halves. Instead your claim check consists of two small envelopes with the same check number written inside each of the two envelopes.

Second, for most claim checks, the numbers are prominently displayed. Your claim check envelopes are sealed until the end of the experiment.

But like other claim check systems, your claim check number differs from anyone else's in the experiment.

The experiment is structured so that when the claim check envelopes are opened they provide no information about your identity. 
Now for the specific steps of the experiment...

(You don't have to memorize the steps; we'll remind you during the experiment.)

\{Each subject in each game reads this slide and the next slide. $\}$ 


\section{Reds Randomly Pick Carriers}

At the beginning of the experiment, the monitors bring a box of large envelopes to the Red participants and shuffle the envelopes.

Each Red participant randomly picks a large envelope from the box.

We call the large envelopes "carriers" because we use them to carry things around.

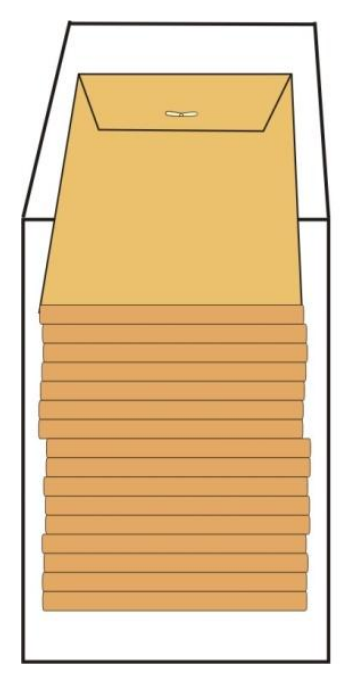

each Red participant randomly picks a carrier from the box 
The next slides are about operationalization.

In the Trapezoid game, from which the following slides are drawn, the slides show the trapezoid diagram and remind subjects how highlighting lines and circling dots determine payments.

In the BDM-like game, the slides show the decision sheet and how to make decisions by filling in the bubbles.

Otherwise, the slides are the same in the two games and are nearly constant. 


\section{Carrier Contents}

The contents of the carrier you picked are:

The trapezoid diagram

Two red envelopes (a) and (b)

Two blue envelopes (c) and (d)

Do not unseal any envelopes until you are told to.

Carrier Contents

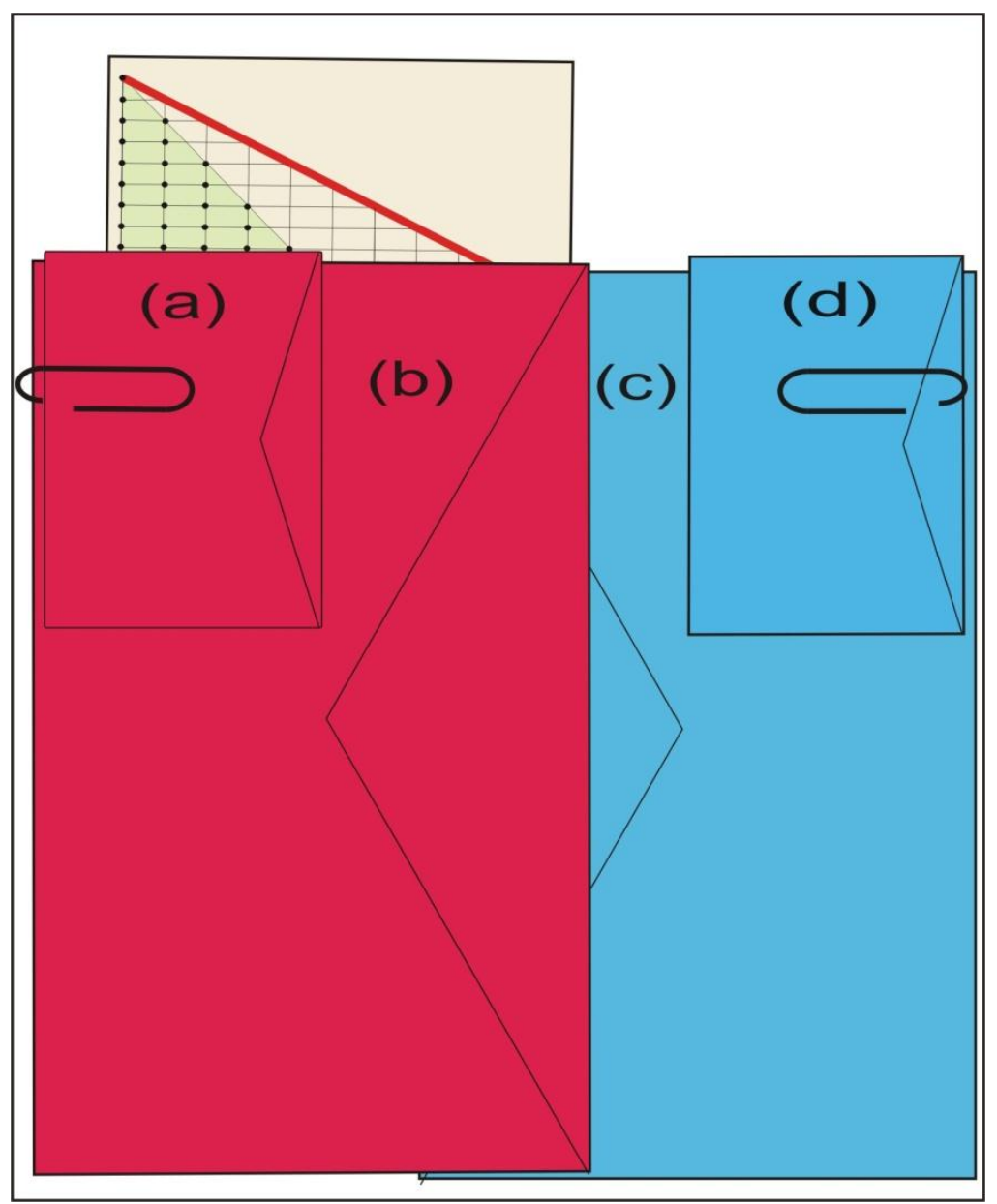




\section{If You Are a Red Participant, Your}

Actions and Decision Are:

First, open the carrier and put the small

Carrier Contents red envelope (a) in your pocket for safe keeping. It contains the top half of your claim check, and you will need it later to get your payment.

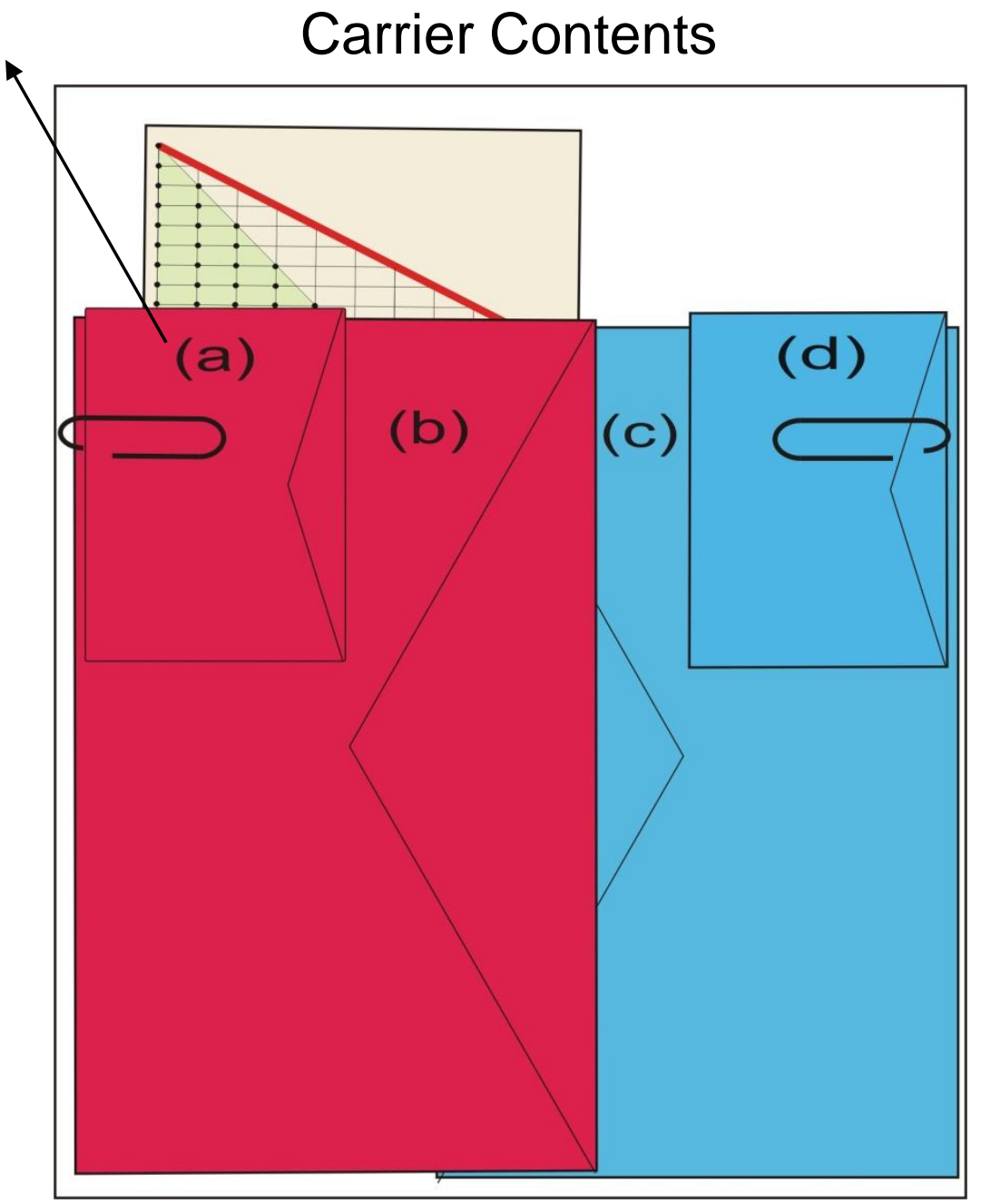




\section{As a Red Participant, Your Actions}

and Decision Are:

Second, take the trapezoid diagram out

Carrier Contents of the carrier and decide which horizontal grid line to choose. Highlight your chosen line with the yellow highlighter. (We don't show the yellow line, because it depends on your choice.)

Later we will explain how you can make your decision private.

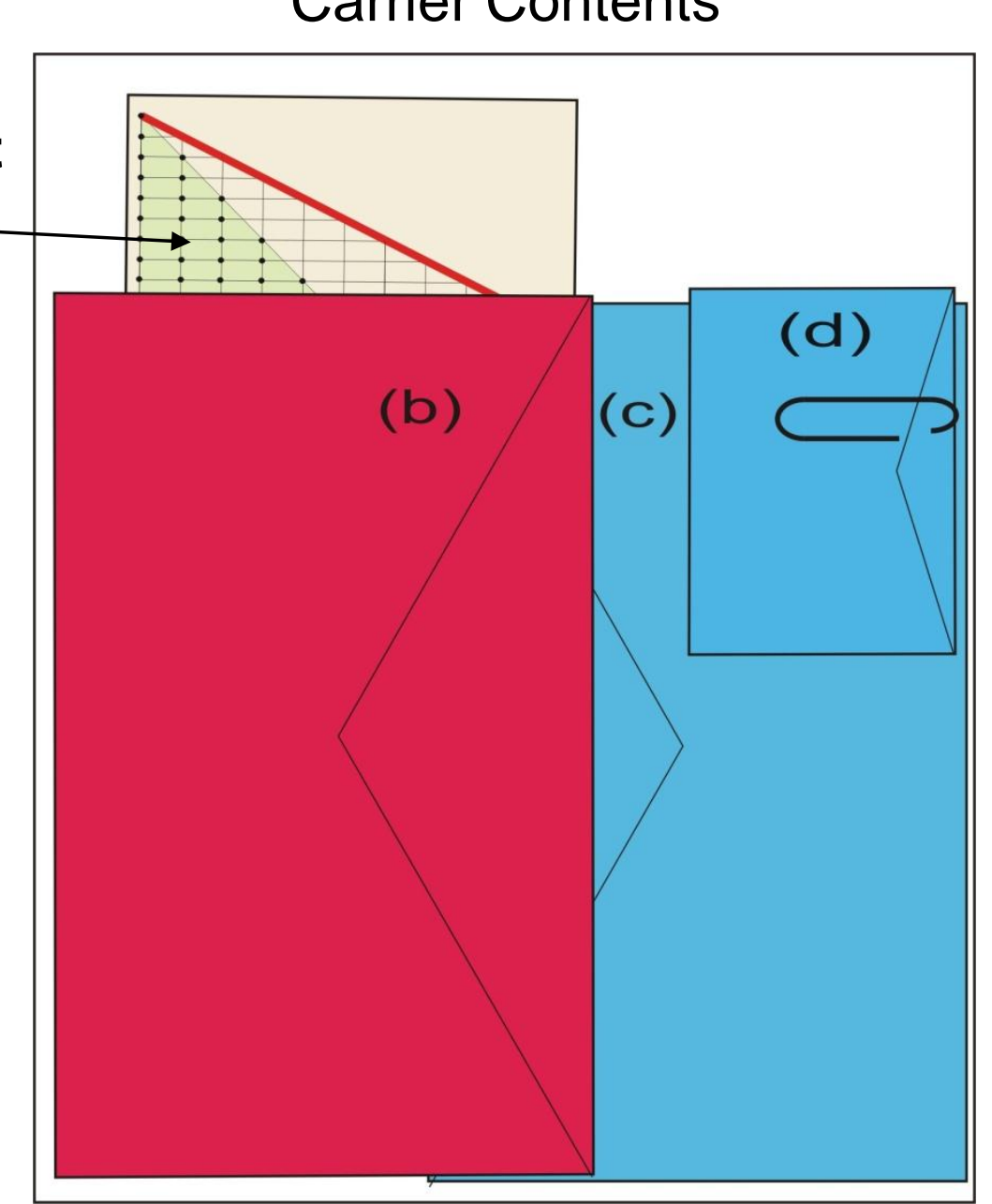




\section{As a Red Participant, Your Actions}

and Decision Are:

Third, put the diagram back in the carrier and make sure that the large envelopes (b) and (c) and the small envelope (d) are in the carrier. Close the carrier.

Carrier Contents

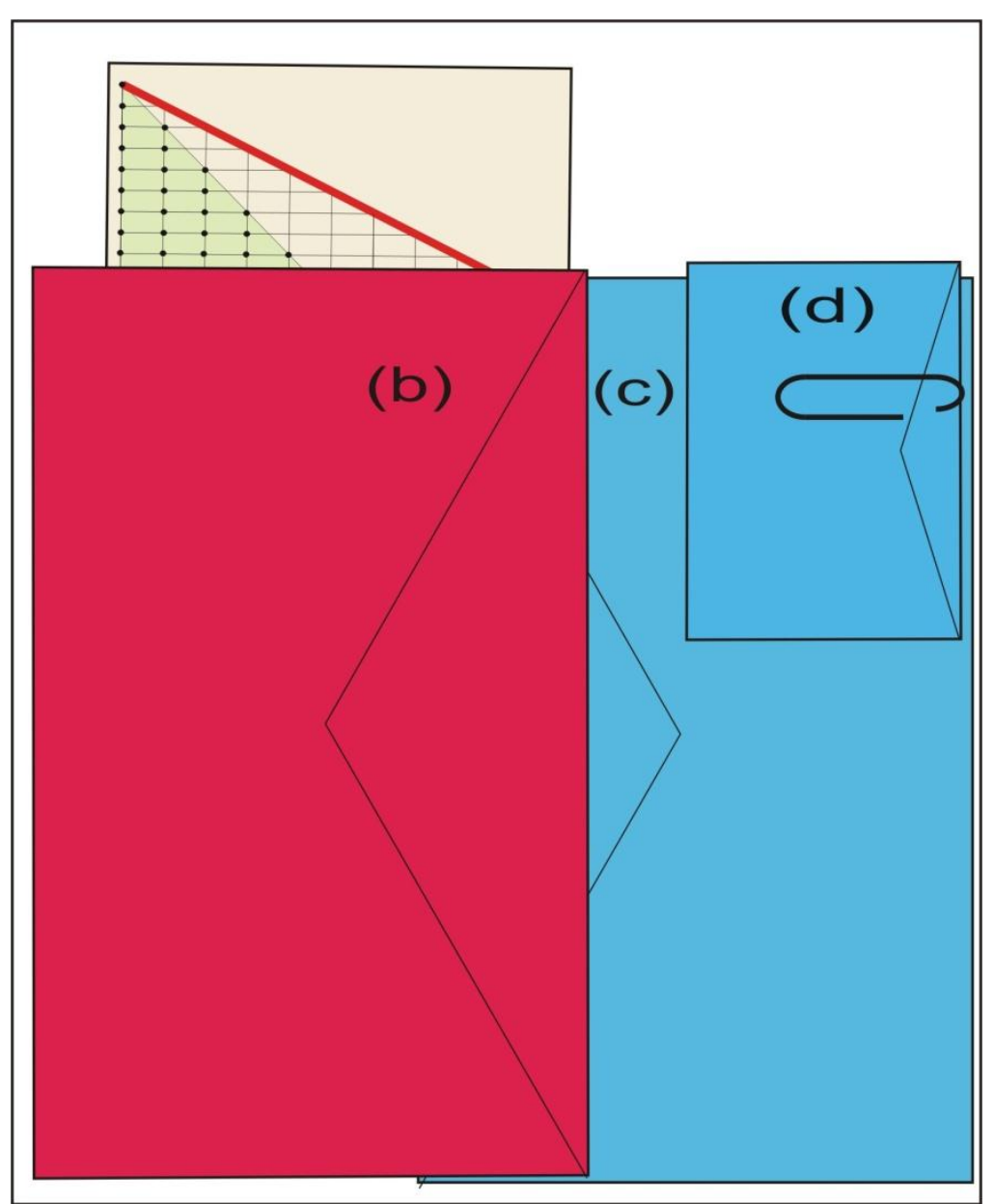




\section{Monitors and Experimenters' Actions}

After all the Reds make their decision the monitors collect the carriers and shuffle them.

The experimenters check the carriers and contents for problems. *

The monitors shuffle the carriers and bring them to the Blues.

${ }^{*}$ Among other things, the experimenters checked that no subject attempted to communicate information to his or her counterpart beyond the permitted highlighting of a line, circling of a dot, or filling in of a bubble. The instructions stated: "Clearly indicate your decision. Make no other marks on your decision sheet. Make no marks on the envelopes or on the carrier. If your decision is not clear, or there are problems of marking envelopes, or the carrier, or its contents, then your decision may not be useable and you may be disqualified from receiving payment." These instructions were strictly followed, which safeguarded anonymity and ruled out transmission of suggestions or requests. 


\section{Blue Participants Randomly \\ Pick Carriers, Establishing Pairs}

Each Blue participant randomly picks a carrier.

With 16 Red and 16 Blue participants,

some (anonymous) Blue participant will pick the carrier containing the decision of the (anonymous) participant we have been referring to as "you."

This anonymous Red participant and this anonymous Blue participant are now paired for the rest of the experiment. 


\section{Carrier Contents}

The contents of the carrier you, the Blue participant, picked are:

Carrier Contents

\section{$\underline{\text { Trapezoid diagram }}$}

One large red envelope (b)

Two blue envelopes (c) and (d)

Do not unseal any envelopes until you are told to.

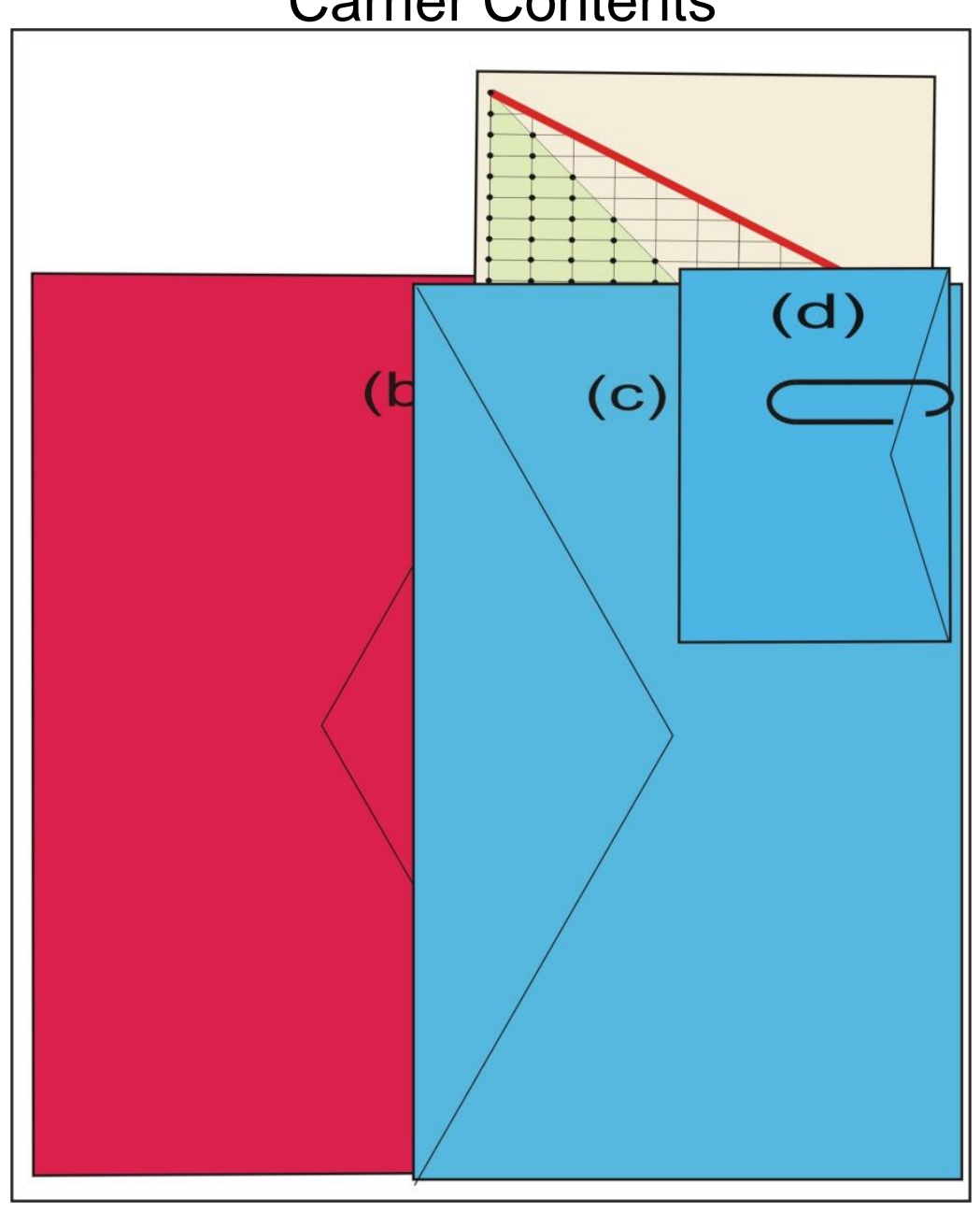




\section{Blue's Action}

First, open the carrier. As the Blue participant of the pair, put the small blue envelope (d) in your pocket for safe keeping. You will need it later to get your payment.

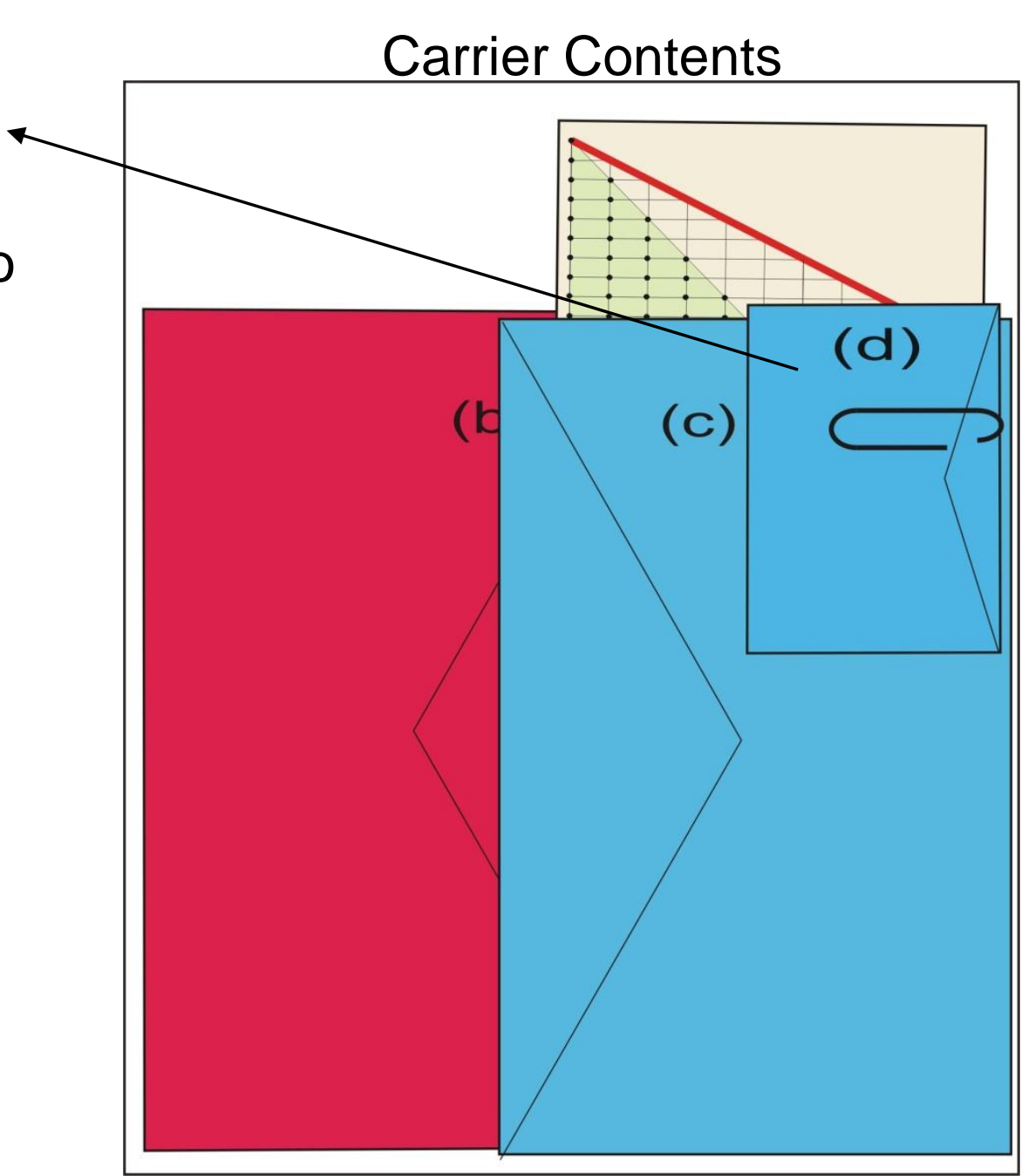




\section{Blue's Actions and Information}

Second, take the trapezoid diagram out of the carrier and learn of Red's decision from the yellow highlight. (Red's choice of a yellow line is not shown in this slide because the chosen line depends on Red's choice).

Carrier Contents

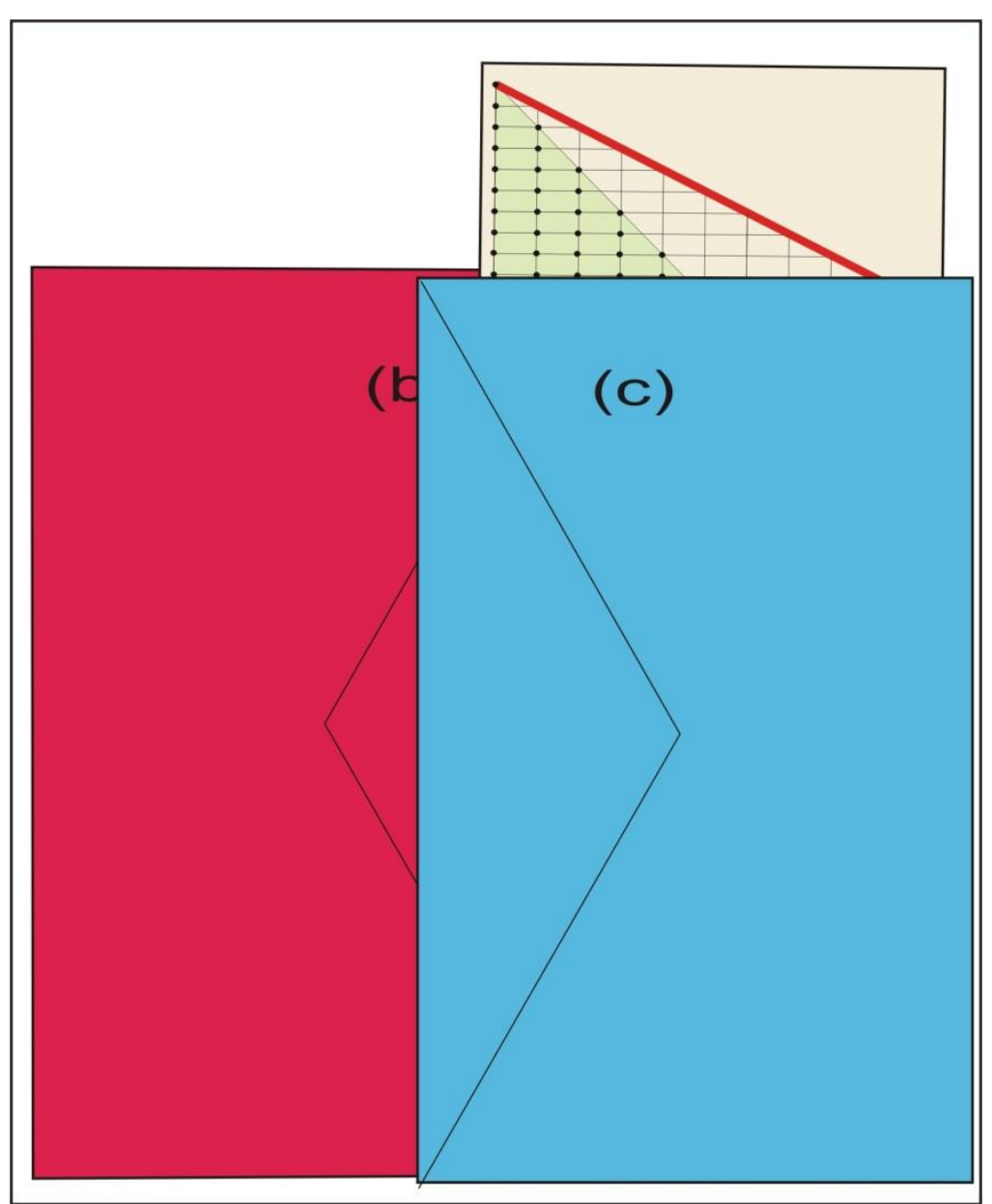




\section{Blue's Actions, Information and Decision}

Third, you make a decision of which dot to choose on the yellow line. You indicate your choice by a circle. (Your choice is not shown in this slide because it depends on Red's and your decision).

Later we will explain how you can make your decision private.

Carrier Contents

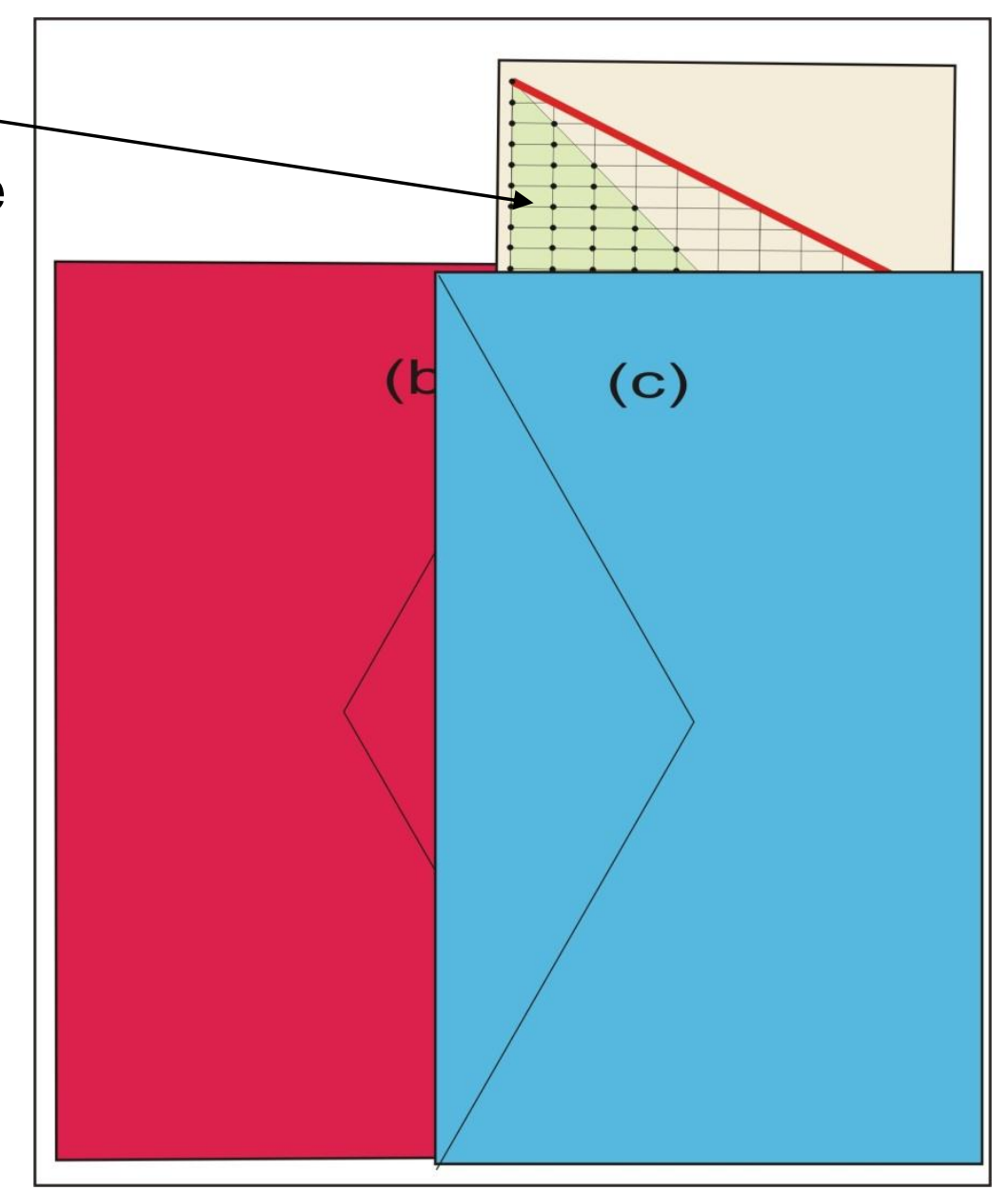




\section{Blue's Actions, Information and Decision}

Fourth, put the diagram back in the carrier and make sure envelopes (b) and (c) are in the carrier. Close the carrier.

Carrier Contents

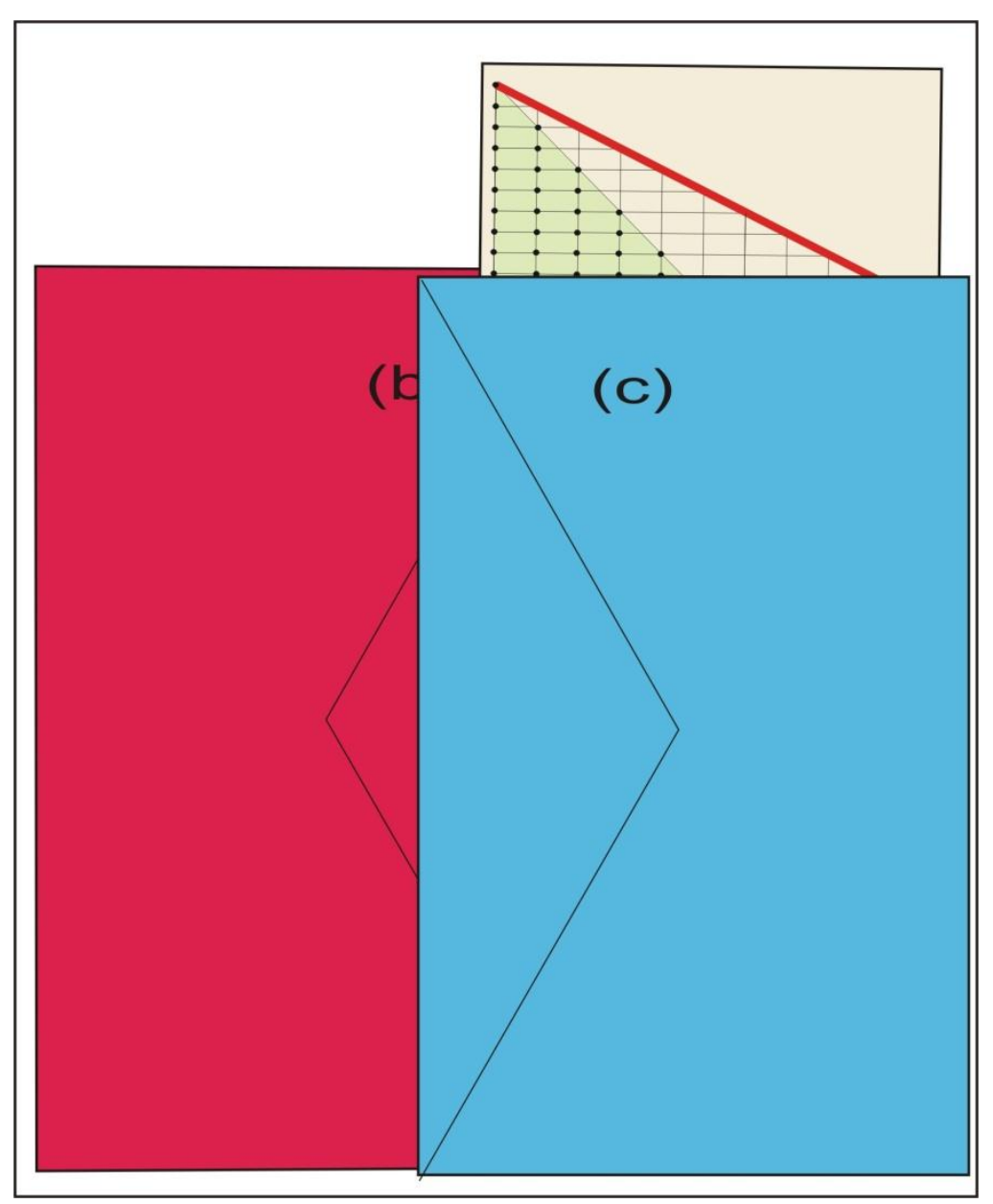


The Experimenters' and Monitors' Actions and Information

After Blues make their decisions, the monitors collect the carriers, shuffle them and deliver them to the experimenters.

The monitors watch the experimenters open the carriers one by one, check for problems, and unseal and discard envelopes (b) and (c). Inside are white payment envelopes to which the sealed bottom halves of Red's and Blue's claim checks were stapled before the experiment began.

The experimenters use the trapezoid diagram and Red's and Blue's decisions (not shown in this slide) to find the correct cash amounts to place in each payment envelope.

Carrier Contents

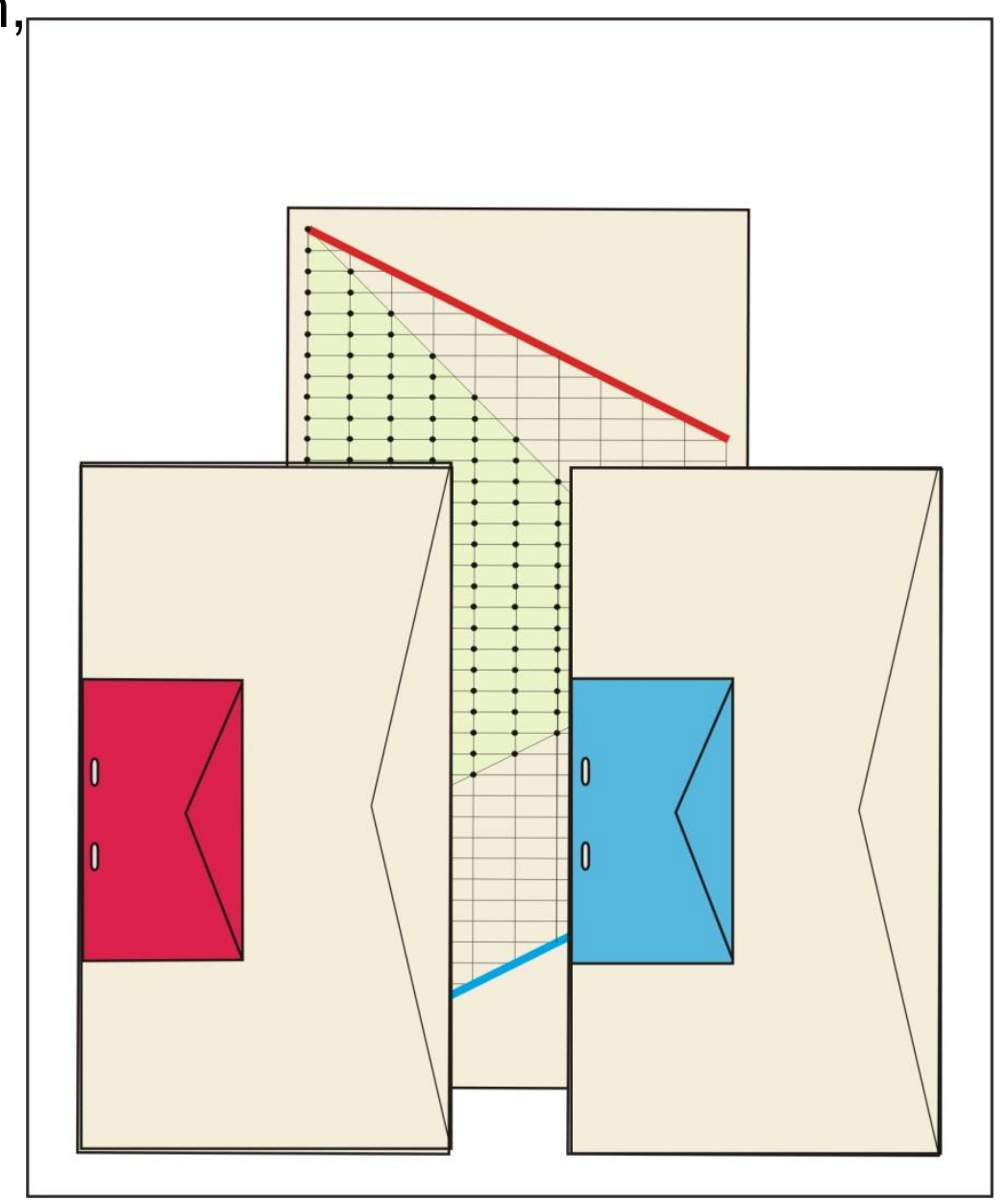




\section{Experimenters' and Monitors' Actions}

The experimenters record the decisions of each anonymous pair of participants, put the amounts in the payment envelopes, and seal these envelopes.

The monitors put the payment envelopes for Reds and Blues in separate piles. The bottom halves of the claim checks remain sealed and stapled to the payment envelopes.

The monitors unseal the bottom halves of the claim checks for Reds at one table, and for Blues at another table. The payment envelopes remain sealed.

Carrier Contents

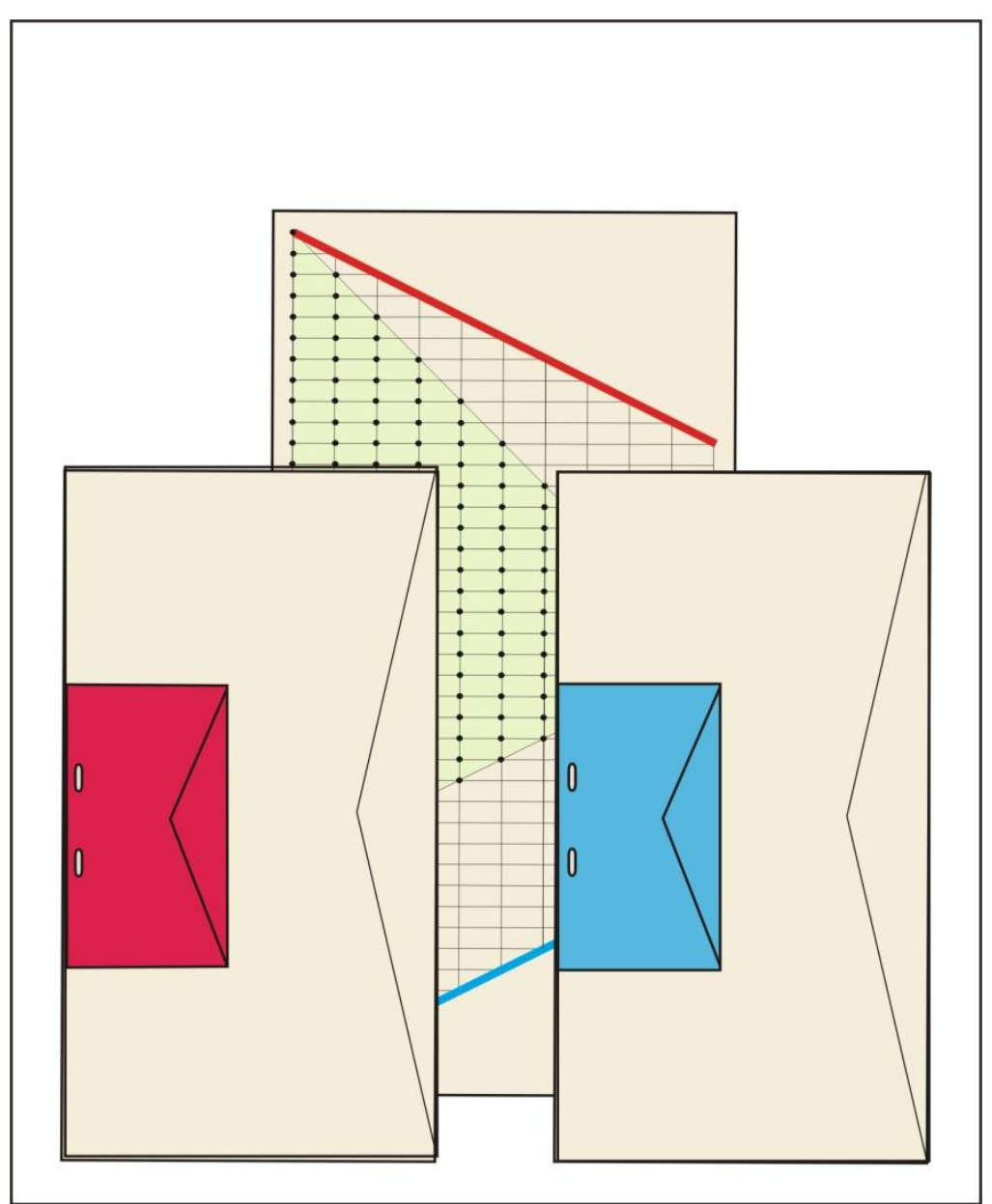


The Experimenters' and Monitors' Actions and Information

The monitors ask participants, one by one, to produce from their pockets the top half of their claim check.

The monitors find the bottom half of the claim check that matches the top half. With the bottom half stapled to the payment envelope, the correct payment is delivered to each of the participants.

Because of the shuffling, the experimenters and monitors do not know the amount in anyone's payment envelope. Privacy is preserved at the same time as the correct payments are made.

Carrier Contents

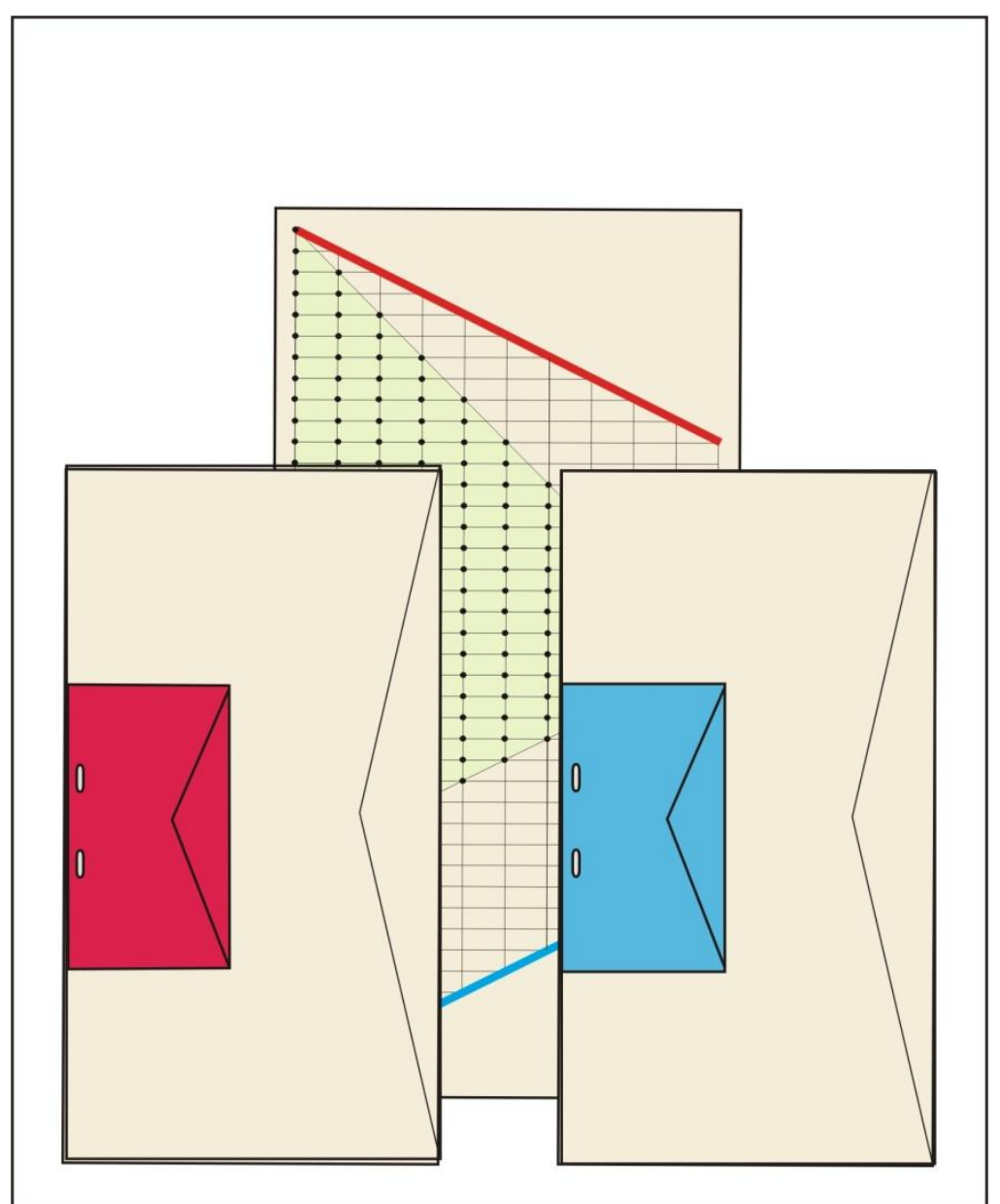


Near the end of the instructions we included four practice exercises to ensure the subjects understood the instructions, then an experimenter began the "for keeps" part of the experiment by reading a script to remind the monitors and subjects of each step required of them, this time without practice exercises. An experimental session took 60 to 80 minutes, including the payments. Thirty-two subjects participated in each experimental session, not counting the monitors.*

To reduce the amount of talk about trust games, we scheduled gaps between the games. We scheduled the Trapezoid game (or experiment) for November 14, 2009, close to Thanksgiving and travel time. We scheduled the BDM-like experiment for December 6, 2009 when the subjects were finishing the semester. Since the 64 subjects playing the Blue and Red roles on Nov. 14 and the 64 playing the corresponding roles on Dec. 6 were drawn from a general student population numbering about 6,000 , it seems very unlikely that any subject in the BDM-like game (or experiment) arrived with detailed knowledge about the Trapezoid game played by an earlier subject, and all but certain that any such word-of-mouth was limited to a handful of individuals at most. It is even less likely that Trapezoid treatment subjects had encountered the conventional, word-based trust game elsewhere, so their likelihood of associating the game we asked them to play with the one played in BDM (1995) would be very small.

* Thirty-two subjects participated in each of the 6 sessions, but in one session a subject misplaced one of her envelopes, invalidating her and her counterpart's data. 


\section{Figure 1. Observed Outcomes}

In the Trapezoid game, 12 Reds sent $\$ 10$, the maximum a Red can send. Another 11 Reds send $\$ 0$, the minimum a Red can send.

In the BDM-like game, 19 Reds sent $\$ 10$, the maximum a Red can send. Another 2 Reds sent $\$ 0$, the minimum a Red can send.
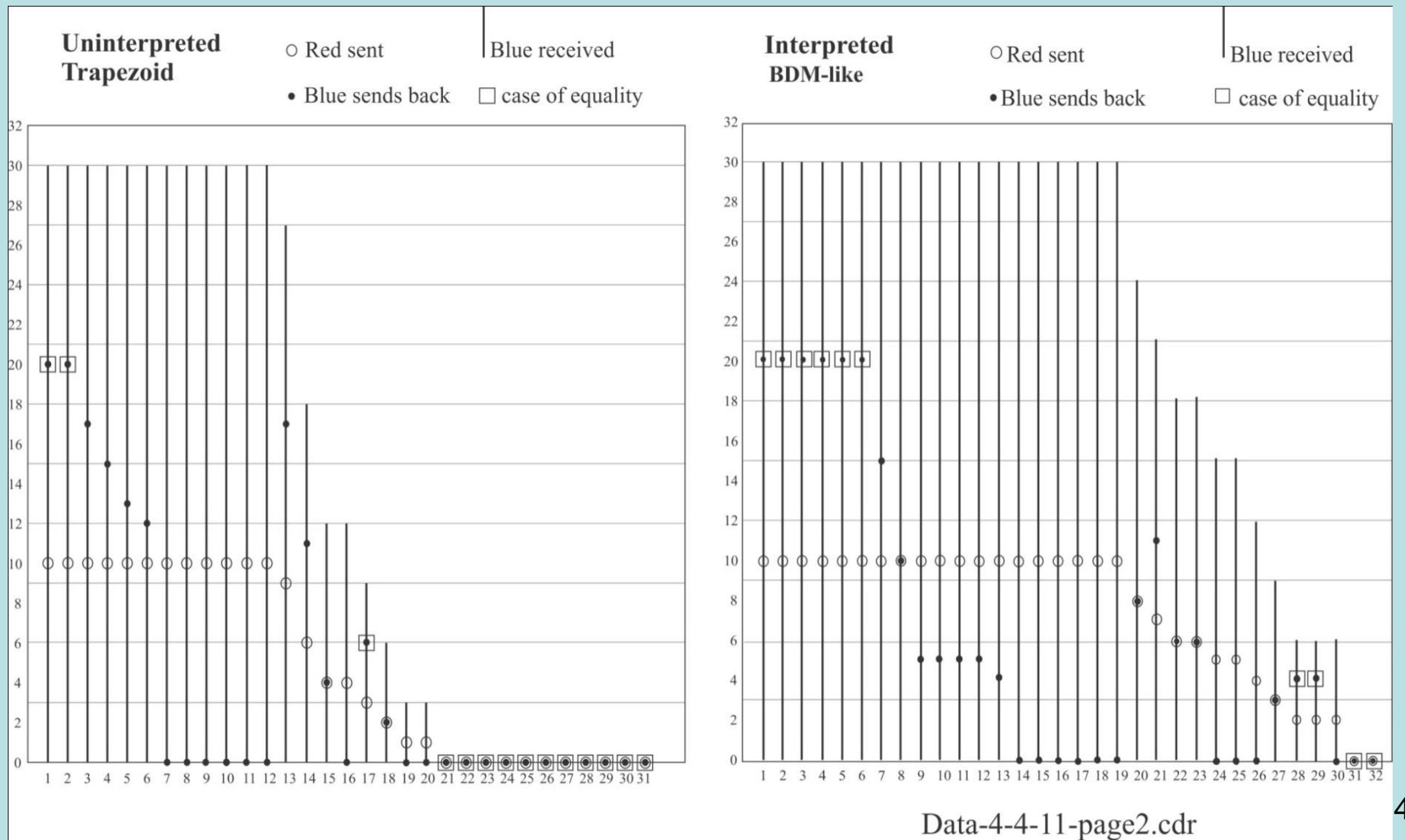


\section{The Correspondence Relation}

The Trapezoid game varies from the BDM-like game in two ways: interpretation and form.

A subject in the Trapezoid game learns the game form with its circles, dots, and payment structure, but not the interpretation of the game.

A subject in the BDM-like game learns the BDM-like game form with its bubbles, payment structure and interpretation in terms of send, etc.

The subjects only observe one game or the other, and do not observe the variations. Seeing both games, the experimenters can see the variations and identify a correspondence relation. Consider an example at the beginning in the Trapezoid game: Red's decision was to choose the third line down (p. 10). In response Blue chose the second dot to the right on Red's chosen line (page 12). In result, Red's payment is $\$ 9$ and Blue's payment is $\$ 15$ (p. 14).

Now consider an example at the beginning in the BDM-like game. Red's decision was to send $\$ 2$ (p. 18). Blue sends back $\$ 1$ (p. 21). In result, Red's payment is $\$ 9$ and Blue's payment is $\$ 15$ (p. 22). It is straight-forward to check that for any Red and Blue decision in the Trapezoid game there is a unique Red and Blue decision in the BDM-like game with the same Red and Blue payments (and for any Red and Blue decision in the BDM-like game there is a unique Red and Blue decision in the Trapezoid game with the same Red and Blue payments). Thus it is possible to translate from lines and dots to "send" and "send back" as we did in Figure 1. 


\section{The Correspondence Relation and the Two Variables}

The correspondence relation shows a similarity between the Trapezoid game and the BDM-like game (the correspondence also extends to the original BDM trust game). Nonetheless, the two design "variations" - interpreted or uninterpreted and spatial or word-based - may lead to differences in behavior.

Spatial games are often uninterpreted and useful when there are questions about an interpreted game, which is our situation, and we designed the Trapezoid game as an uninterpreted spatial game. In contrast the BDM-like game is interpreted and is not spatial. 


\section{Recall Two Observations in Figure 1 and Two Questions}

12 cases of maximum trust in the Trapezoid game and

$\mathbf{1 9}$ cases of maximum trust in the BDM-like game

Does this suggest that value laden words have an effect on behavior? Is the difference in the small number of cases statistically significant?

The BDM-like game used the words "show up fee" (possibly suggesting entitlement), "send" and "send back" (possibly suggesting reciprocity), and "each dollar sent ... will be tripled" (possibly suggesting the experimenters want the money to be sent).

In contrast, in the Trapezoid game we know for sure that the above words will not increase reciprocity or have other effects for the simple reason that the above words are completely absent in the Trapezoid game.

These and other differences between the Trapezoid and BDM-like games provide a way of testing for the effects of the possibly suggestive meanings that are the basis of the doubts we started with. 


\section{The Fisher Exact Test}

The Fisher exact test has advantages: It works with small data sets, is based on an exact calculation (not an approximation or an asymptote), and apparently was the genesis of $p$-values. For many years the National Cancer Institute used the Fisher exact test to evaluate the toxicity of chemicals, e.g. by exposing a treated group of rats to a possibly toxic chemical while a control group, kept under otherwise identical conditions, was not exposed to it.

The qualitative results reported below - that is, whether particular differences are statistically significant at the $1 \%$ level, at the $10 \%$ level, or at neither of those levels - hold also for the chi-square and other tests.

We used one-tailed tests because it appears that the possibly value laden words would either increase the effect on the words or would have no effect at all. It also appears that the effect of transparency and neutrality would be minor or none in respect to the words.

Each of the four $2 \times 2$ tables in Figure 2 provides a step toward resolving or confirming the doubts we started with. We refer to each table by its first row heading. 


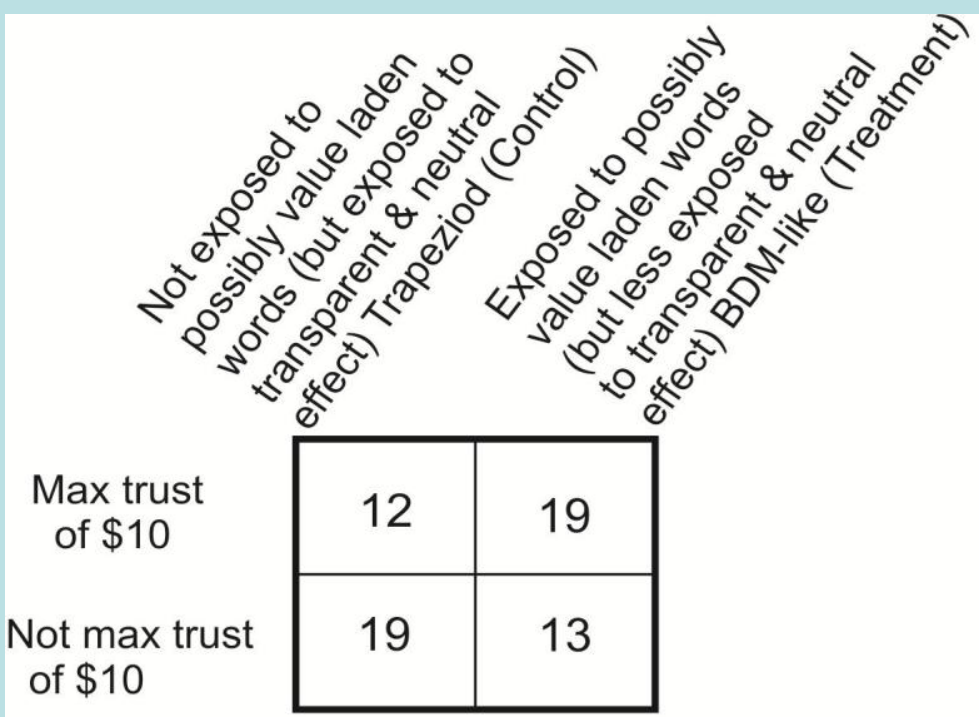

$p$-value 0.082

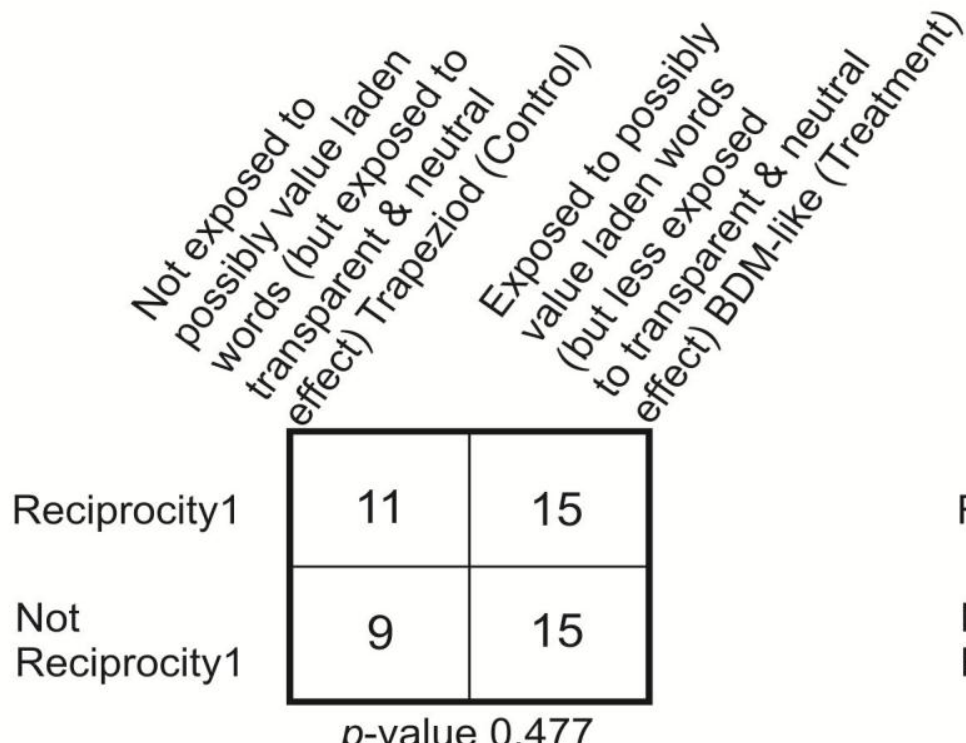

A case of recipocity1 happens when an anonymous Red has sent a positive amount to Blue and Blue sends back as much or more than the paired Red sent to Blue

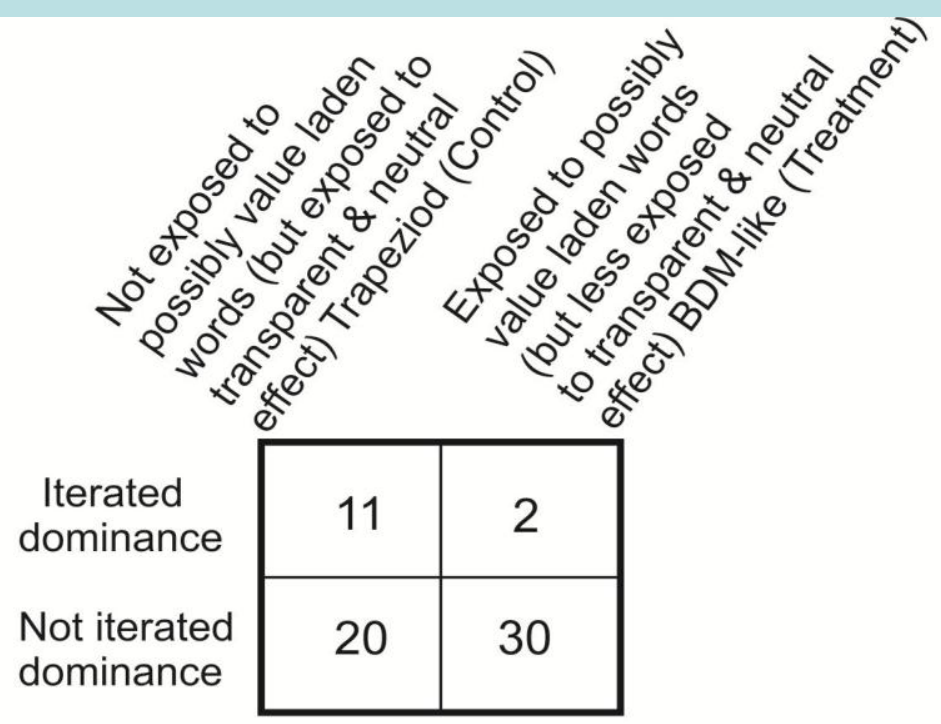

p-value 0.004

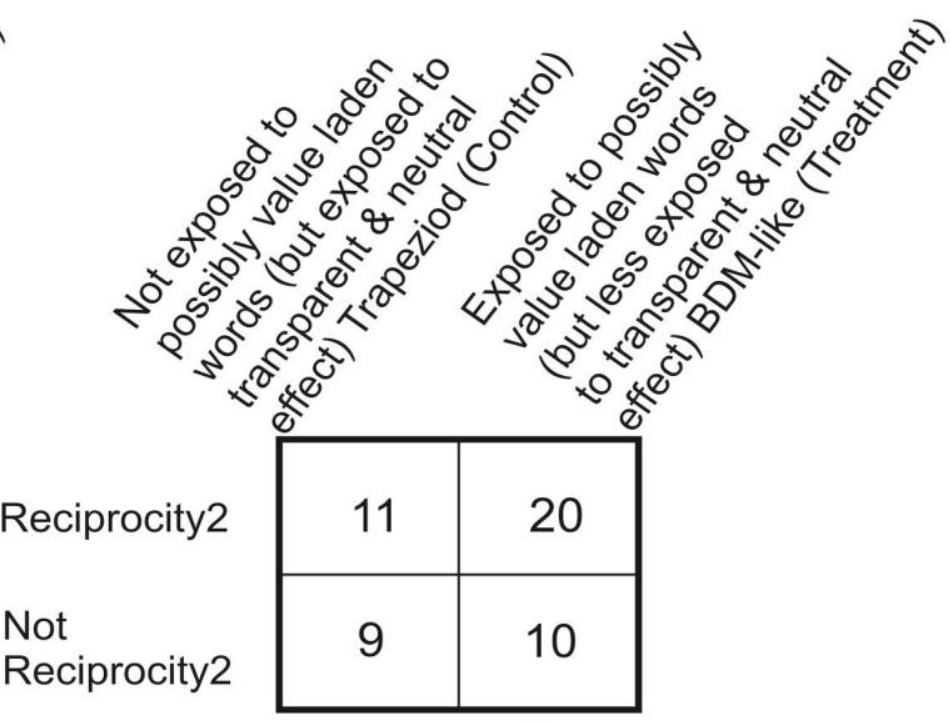

$p$-value 0.220

A case of recipocity2 happens when an anonymous Red has sent a positive amount to Blue and Blue sends back any positive amount to the paired Red
Figure 2. $2 \times 25$ tables corel-trust-game 4 


\section{"Max trust of \$10," Trapezoid Game}

The left column of the Max trust table shows decisions about "maximum trust" made by subjects in the Trapezoid game.

The top of the left column shows the number 12, which is the number of Reds in the Trapezoid game who decided to highlight the lowest line in the Trapezoid diagram (or each of 12 Reds "sent" $\$ 10$ each to an anonymous Blue).

The bottom of the left column shows the number 19, the number of Reds in the Trapezoid game who decided not to highlight the lowest line in the Trapezoid diagram (or each of 19 Reds did not "send" \$10 to an anonymous Blue).

Reds who highlighted the lowest line trusted the most, and could end up with a payment of $\$ 0$ when they could have had a guaranteed payment of $\$ 10$; Reds who did not highlight the lowest line guaranteed their payment would be at least more than $\$ 0$.

No subject was exposed to the possibly value laden words "show-up fee," "send," "send back," or "each dollar sent ... will be tripled" which are absent in the Trapezoid game. "Trust" and "guarantee" are used in this commentary but absent in the two games. In this sense, the Trapezoid game acts as a Control.

Possibly in the Trapezoid game some subjects had the strategic idea, rightly or wrongly, that giving the generous maximum would elicit enough reciprocity to make it worthwhile. Or some subjects anticipated the feeling of well-being from oxytocin. Or some subjects brought their values to the lab, including trust. Such motivations might explain some of the observed behavior (that is, the behavior of the 12 Reds who sent the maximum amount, or the behavior of the 19 Reds who sent less or maybe nothing). 


\section{"Max trust of \$10," BDM-like Game}

The right column of the table shows decisions about "maximum trust" made by subjects in the BDM-like game.

The top of the right column shows the number 19, which is the number of Reds in the BDM-like game who decided to fill in the highest bubble available (\$10) for Reds in the BDM-like game.

The bottom of the right column shows the number 13 , which is the number of Reds in the BDM-like game who each decided to not send his/her entire money available (\$10) to an anonymous Blue; these Reds guaranteed their payment would be at least more than $\$ 0$.

The 19 Reds in the BDM-like game who sent the most (\$10) trusted the most; these 19 Reds could end up with a payment of $\$ 0$ when they could have guaranteed themselves $\$ 10$.

In the BDM-like game every subject was exposed to the possibly value laden words. In this sense, the BDM-like game acts as a Treatment, in respect to the words.

In addition to the Treatment with words, the same motivations operating in the Trapezoid Game (i.e., the strategic idea and anticipated well-being from oxytocyn), might explain some trusting also in the BDM-like game. 


\section{"Max trust of \$10," Comparison}

In the Trapezoid game the frequency of Reds choosing maximum trust was $12 /(12+19)=\mathbf{0 . 3 9}$.

In the BDM-like game the frequency of Reds choosing maximum trust was $19 /(19+13)=\mathbf{0 . 5 9}$.

The difference in frequencies between 0.39 without the words in the Trapezoid game, and 0.59 with the words in the BDM-like game is significant ( $p$-value 0.082).

This difference in frequency suggests that the value laden words had an effect in increasing the number of subjects giving the maximum $\$ 10$. 


\section{"Iterated Dominance," Trapezoid Game}

The left column shows decisions about "Iterated Dominance" made by subjects in the Trapezoid game.

The top of the left column shows the number 11, which is the number of Reds in the Trapezoid game who decided to highlight the highest line in the Trapezoid diagram (at the top of the triangle). This is the case of Iterated Dominance in the game in which Blue has no decision to make (there is only one dot at the top of the triangle for Blue to choose from). In Figure 1 (p. 45), a case of Iterated Dominance is shown as a circle on the horizontal axis with a dot on the axis and square also on the horizontal axis (this means a Red sends $\$ 0$ to Blue), and Red and Blue each get $\$ 10$ equally.

The bottom of the left column shows the number 20, the number of Reds in the Trapezoid game who decided not to highlight the highest line in the Trapezoid diagram. These are not cases of Iterated Dominance. A Red who sent $\$ 10$ to a Blue takes a risk. If Blue sends $\$ 20$ back, then Red and Blue each end up with $\$ 20$ (there were two cases of that, shown by cases 1 and 2, with the squares signifying equality at the $\$ 20$ mark). But if Blue sends back $\$ 0$, Red gets $\$ 0$ and Blue gets $\$ 40$ (there were 6 cases of that outcome, see cases 7-12). 


\section{“Iterated Dominance," Trapezoid Game (cont.)}

It is well-known that spatial games tend to be value neutral and transparent. For example, in the Trapezoid game it was easy to visualize payments as distances. It was also easy to explain the Trapezoid game without using the words "send," "send back," "show-up fee," and "triple." Up until now we have focused on the absence of such words in the Trapezoid game in its role as a Control.

We now consider how the Trapezoid game's characteristics of neutrality and transparency related to Iterated Dominance. We find that a Blue can easily see that his/her best (monetary) strategy is to circle the left-most dot, whatever line Red chooses. Realizing this, Red's best (monetary) strategy is to choose the top line. That is, it is easy for a Red to see Iterated Dominance as a solution concept.

As already noted, when a Red chooses the top line, Red ensures a case of Iterated Dominance, because when Red chooses the top line Blue has no decision to make. In our example when Red forces Iterated Dominance, Red avoids considerable risk and assures a guaranteed outcome to herself and less benefit for Blue.

The 11 cases of Iterated Dominance are not surprising in light of the Trapezoid game's transparency and moral neutrality (and simplicity of this version of Iterated Dominance). 


\section{"Iterated Dominance," BDM-Like Game}

The right column shows decisions about "Iterated Dominance" made by subjects in the BDM-like game.

The top of the right column shows the number $\mathbf{2}$, which is the number of Reds in the BDM-like game who decided to fill the bubble labeled 0 . This is the case of Iterated Dominance in the game in which Blue has no decision to make. In Figure 1, a case of Iterated Dominance is shown as a circle on the horizontal axis with a dot on the axis and square also on the horizontal axis (this means a Red sends $\$ 0$ to Blue), and Red and Blue each get $\$ 10$ equally. This works via the correspondence relation.

The bottom of the right column shows the number 30, the number of Reds in the BDM-like game who decided to fill a bubble which is Not 0 . 


\section{“Iterated Dominance," Comparison}

The $\mathbf{1 1}$ cases of Iterated Dominance in the Trapezoid game compared with the $\mathbf{2}$ cases of Iterated Dominance in the BDM-like game is not very surprising. The characteristics of neutrality and transparency are more salient in the Trapezoid game than in the BDM-like game.

Trapezoid frequency of Reds choosing Iterated Dominance was $11 /(11+20)=0.35$. BDM-like frequency of Reds choosing Iterated Dominance was $2 /(2+30)=0.06$.

This difference in frequencies between the Trapezoid and BDM-like games is significant ( $p$-value 0.004). 


\section{"Reciprocity1," Trapezoid Game}

The left column of the table shows decisions about "Reciprocityl" made by subjects in the Trapezoid game.

The top of the left column shows 11, the number of cases of Reciprocity1.

The bottom of the left column shows $\mathbf{9}$, the number of cases of Not Reciprocity1.

Note that the sum of the cases of Reciprocity1 plus Not Reciprocity1 is 20, because 11 Blues could not make a decision due to the 11 cases of iterated dominance (and the disqualified pair, from the missing envelope in the Trapezoid game).

The definition of Reciprocity1 is shown in Figure 1. Restated, a case of Reciprocity1 happens when an anonymous Red has sent a positive amount to a Blue and Blue sends back as much or more than the paired Red sent to Blue. (For example, if Red sends Blue $\$ 5$ and Blue sends back $\$ 8$, then this is a case of Reciprocity1. If Red sends Blue $\$ 8$ and Blue sends back $\$ 5$, then this is not a case of Reciprocity1.)

In the Trapezoid game, No subject was exposed to the possibly value laden words "show-up fee," "send," "send back," or "each dollar sent ... will be tripled," these words are absent in the Trapezoid game. In this sense, the Trapezoid game acts as a Control.

With 11 cases of Reciprocity 1 and 9 cases of Not reciprocity1, the frequency of Reciprocity1 is $11 /(11+9)=\mathbf{0 . 5 5}$, meaning that Blues reciprocated (Reciprocity1) in a little more than half the cases in which Blue could make a decision. 


\section{"Reciprocity1," BDM-like Game}

The right column of the table shows decisions about "Reciprocity1" made by subjects in the BDM-like game.

The top of the left column shows $\mathbf{1 5}$, the number of cases of Reciprocity1.

The bottom of the left column shows $\mathbf{1 5}$, the number of cases of Not Reciprocity1.

Note that the sum of the cases of Reciprocity1 plus Not Reciprocity1 is 30 , because 2 Blues could not make a decision due to the 2 cases of iterated dominance).

The definition of Reciprocity1 is the same as in the previous slide.

In the BDM-like game, Every subject was exposed to the possibly value laden words and in the BDM-like game the words act as a Treatment.

With 15 cases of Reciprocity 1 and 15 cases of Not reciprocity 1 , the frequency of reciprocity is $15 /(15+15)=\mathbf{0 . 5 0}$, meaning that Blues reciprocated (Reciprocity1) in half the cases in which Blue could make a decision. 


\section{"Recipocity1," Comparison}

In the Trapezoid game there were: $\mathbf{1 1}$ cases of Reciprocity 1 and $\mathbf{9}$ cases of Not Reciprocity1, with a frequency $11 /(11+9)=\mathbf{0 . 5 5}$.

In the BDM-like game there were: $\mathbf{1 5}$ cases of Reciprocity1 and $\mathbf{1 5}$ cases of Not reciprocity1, with a frequency $15 /(15+15)=\mathbf{0 . 5 0}$.

The difference in frequencies between $\mathbf{0 . 5 5}$ without the words in the Trapezoid game, and $\mathbf{0 . 5 0}$ with the words in the BDM-like game is insignificant ( $p$-value 0.477).

This negative finding suggests that the possibly value laden words in the lab have little or no effect on reciprocity (Reciprocity1).

Note that we found evidence that possibly value laden words have an effect in increasing the number of subjects giving the maximum $\$ 10$. And now we find evidence that, under different circumstances, words have little or no effect. 


\section{"Reciprocity2," Trapezoid Game}

The left column of the table shows decisions about "Reciprocity2" made by subjects in the Trapezoid game.

The top of the left column shows 11, the number of cases of Reciprocity2.

The bottom of the left column shows 9, the number of cases of Not Reciprocity2.

Note that the sum of the cases of Reciprocity 2 plus Not Reciprocity 2 is 20 , because 11 Blues could not make a decision due to the 11 cases of iterated dominance (and the disqualified pair, from the missing envelope in the Trapezoid game).

In a more lenient definition of reciprocity, a case of Reciprocity2 "happens when an anonymous Red has sent a positive amount to a Blue and Blue sends back as much or more than the paired Red sent to Blue." (For example, if Red sends Blue $\$ 8$ and Blue sends back $\$ 5$, then this is a case of Reciprocity2. If Red sends Blue $\$ 8$ and Blue sends back $\$ 0$, then this is not a case of Reciprocity2.)

In the Trapezoid game, No subject was exposed to the possibly value laden words. In this sense, the Trapezoid game acts as a Control.

With 11 cases of Reciprocity 2 and 9 cases of Not Reciprocity2, the frequency of reciprocity is $11 /(11+9)=\mathbf{0 . 5 5}$, meaning that Blues reciprocated (Reciprocity 2 ) in a little more than half the cases in which Blue could make a decision. 


\section{"Reciprocity2," BDM-like Game}

The right column of the table shows decisions about "Reciprocity2" made by subjects in the BDM-like game.

The top of the right column shows 20, the number of cases of Reciprocity2.

The bottom of the right column shows $\mathbf{1 0}$, the number of cases of Not Reciprocity2.

The definition of Reciprocity 2 is the same as in the previous slide.

In the BDM-like game, Every subject was exposed to the possibly value laden words and in the BDM-like game the words act as a Treatment.

With $\mathbf{2 0}$ cases of Reciprocity 2 and $\mathbf{1 0}$ cases of Not Reciprocity2, the frequency of reciprocity is $20 /(20+10)=\mathbf{0 . 6 7}$, meaning that Blues reciprocated (Reciprocity2) in two-thirds the cases in which Blue could make a decision.*

* Note that among Trapezoid game subjects, no Blue subject satisfied Reciprocity2 without also satisfying Reciprocity1, so the numbers on the left sides of both tables are the same. The same is not true, however, for BDM-like game subjects. 


\section{"Recipocity2," Trapezoid \& BDM-like (comparison)}

In the Trapezoid game there were: $\mathbf{1 1}$ cases of Reciprocity 2 and $\mathbf{9}$ cases of Not Reciprocity2, with a frequency $11 /(11+9)=\mathbf{0 . 5 5}$.

In the BDM-like game there were: $\mathbf{2 0}$ cases of Reciprocity 2 and $\mathbf{1 0}$ cases of Not Reciprocity2, with a frequency $20 /(20+10)=\mathbf{0 . 6 7}$.

The difference in frequencies between $\mathbf{0 . 5 5}$ without the words in the Trapezoid game, and $\mathbf{0 . 6 7}$ with the words in the BDM-like game is insignificant ( $p$-value 0.220 ).

This negative finding suggests that the possibly value laden words in the lab have little or no effect on reciprocity (Reciprocity2).

Note that using Reciprocity 2 we found slightly less negative evidence that the words have little or no effect.*

* Possibly the presence of the words "send back" help to account for the presence of 5 cases in which Blue sent back a small positive amount in the BDM-like game versus the absence of such cases in the Trapezoid game. 


\section{Results and Conclusions}

Could the language of "send," "send back" be the source of reciprocal behavior in trust games? Could efforts to achieve double-blind anonymity be a source of interpretation (eg."it's okay to be selfish" or "the experimenters want us to act selfishly")?

We wanted to design an experimental trust game that was uninterpreted or nearly so. This quest took us four years, not long for an experiment, but down paths that surprised us.

(1) Our evidence does not support the conjecture that "social" behaviors are present in the trust game solely because they are induced by verbal cues. See pp. 51, 53, 54, $55,57,58,60,61,63$.

(2) Evidence of the effect of value laden words, compared to without the words, in pp. 50 - 53. Max trust of $\$ 10$.

(3) Evidence of neutrality and transparency in pp. 50 and $54-57$. Iterated Dominance.

(4) Evidence of the possibly value laden words, having little or no effect on reciprocity in pp. 50 and $58-63$. Recipocityl \& Recipocity2. 


\section{Contributions that Made the Results Possible}

(a) The design of the Trapezoid game compared with the BDM-like game. And more generally uninterpreted spatial games.

(b) The design of a double-blind procedure, simple and symmetric, based on claim checks.

(c) The correspondence relation.

(d) Iterated dominance as a solution concept.

(e) Increased use of graphics, as a research tool. 


\section{References}

Berg, Joyce, John Dickhaut, and Kevin McCabe, 1995, "Trust, Reciprocity and Social History," Games and Economic Behavior 10: 122-42.

Kosfeld, Michael, Markus Heinrichs, Paul Zak, Urs Fischbacher and Ernst Fehr, 2005, "Oxytocin Increases Trust in Humans," Nature 435: 673-676.

Page, Talbot and Louis Putterman, 2011, Experiment Instructions for Uninterpreted \& Interpreted Trust Games, http://www.econ.brown.edu/fac/Louis Putterman/instructions.html.

Wilson, Rick and Catherine Eckel, forthcoming, "Trust and Social Exchange," in J. Druckman, D. Green, J. Kuklinski and A. Lupia, Handbook of Experimental Political Science. N.Y.: Cambridge University Press. 